\title{
$\Gamma$-CONVERGENCE, SOBOLEV NORMS, AND BV FUNCTIONS
}

\section{HOAI-MINH NGUYEN}

\section{Abstract}

We prove that the family of functionals $\left(I_{\delta}\right)$ defined by

$$
I_{\delta}(g)=\iint_{\substack{\mathbb{R}^{N} \times \mathbb{R}^{N} \\|g(x)-g(y)|>\delta}} \frac{\delta^{p}}{|x-y|^{N+p}} d x d y, \quad \forall g \in L^{p}\left(\mathbb{R}^{N}\right),
$$

for $p \geq 1$ and $\delta>0$, $\Gamma$-converges in $L^{p}\left(\mathbb{R}^{N}\right)$, as $\delta$ goes to zero, when $p \geq 1$. Hereafter || denotes the Euclidean norm of $\mathbb{R}^{N}$. We also introduce a characterization for bounded variation (BV) functions which has some advantages in comparison with the classic one based on the notion of essential variation on almost every line.

\section{Introduction}

Recently, the following new characterization of Sobolev spaces was established in [13, Theorem 2] and [4, Theorem 1].

THEOREM 1

Let $N \geq 1,1<p<+\infty$, and $g \in L^{p}\left(\mathbb{R}^{N}\right)$. Then $g \in W^{1, p}\left(\mathbb{R}^{N}\right)$ if and only if

$$
\varliminf_{\delta \rightarrow 0_{+}} I_{\delta}(g)<+\infty
$$

Moreover,

$$
\lim _{\delta \rightarrow 0_{+}} I_{\delta}(g)=\frac{1}{p} K_{N, p} \int_{\mathbb{R}^{N}}|D g|^{p} d x, \quad \forall g \in W^{1, p}\left(\mathbb{R}^{N}\right),
$$

where $K_{N, p}$ is given by

$$
K_{N, p}=\int_{\mathbb{S}^{N-1}}|e \cdot \sigma|^{p} d \sigma
$$

for any $e \in \mathbb{S}^{N-1}$.

DUKE MATHEMATICAL JOURNAL

Vol. 157, No. 3, (C) 2011 DOI 10.1215/00127094-1272921

Received 5 August 2009. Revision received 21 June 2010.

2010 Mathematics Subject Classification. Primary 28A20; Secondary 26A24, 26 A84.

495 
We recall that when $p=1$ :

(a) $\quad$ if $g \in L^{1}\left(\mathbb{R}^{N}\right)$ and $\underline{\lim }_{\delta \rightarrow 0_{+}} I_{\delta}(g)<+\infty$, then $g \in \mathrm{BV}\left(\mathbb{R}^{N}\right)$ (see [4], [16]);

(b) there exists $g \in W^{1,1}(\mathbb{R})$ such that $\lim _{\delta \rightarrow 0_{+}} I_{\delta}(g)=+\infty$ (example communicated to us by A. Ponce, 2005; see [13]).

This characterization is distinct from the one of Bourgain, Brezis, and Mironescu [2] (see also [6]), but it is inspired by the results of [2]. Quantities similar to $I_{\delta}$ appear in new estimates for the degree (see [3], [15], [7], [9]). Further results related to Theorem 1 are presented in [16] and in recent work of Chiron [10]. In [17], some results in the spirit of the Poincaré inequality and the Sobolev inequality, where $\int_{\mathbb{R}^{N}}|\nabla g|^{p}$ is replaced by $I_{\delta}(g)$, are established.

Let $p \geq 1$ and $N \geq 1$. Define, for $g \in L^{p}\left(\mathbb{R}^{N}\right)$,

$$
J(g)= \begin{cases}\frac{1}{p} K_{N, p} \int_{\mathbb{R}^{N}}|D g|^{p} d x & \text { if } p>1 \text { and } g \in W^{1, p}\left(\mathbb{R}^{N}\right) \\ & \left(\text { resp., } p=1 \text { and } g \in \mathrm{BV}\left(\mathbb{R}^{N}\right)\right), \\ +\infty & \text { otherwise. }\end{cases}
$$

A natural question raised by $\mathrm{H}$. Brezis (personal communication, 2006) is whether $\left(I_{\delta}\right)$ $\Gamma$-converges in $L^{p}\left(\mathbb{R}^{N}\right)$ to $J$ in the sense of De Giorgi for $p>1$ (see, e.g., [5], [11] for an introduction of $\Gamma$-convergence). We recall that a family $\left(I_{\delta}\right)_{\delta \in(0,1)}$ of functionals defined on $L^{p}\left(\mathbb{R}^{N}\right) \Gamma$-converges in $L^{p}\left(\mathbb{R}^{N}\right)(p \geq 1)$, as $\delta$ goes to zero, to a functional $I$ defined on $L^{p}\left(\mathbb{R}^{N}\right)$ if and only if the following two conditions are satisfied.

(G1) For each $g \in L^{p}\left(\mathbb{R}^{N}\right)$ and for every family $\left(g_{\delta}\right)_{\delta \in(0,1)} \subset L^{p}\left(\mathbb{R}^{N}\right)$ such that $g_{\delta}$ converges to $g$ in $L^{p}\left(\mathbb{R}^{N}\right)$ as $\delta$ goes to zero, one has

$$
\varliminf_{\delta \rightarrow 0} I_{\delta}\left(g_{\delta}\right) \geq I(g)
$$

(G2) For each $g \in L^{p}\left(\mathbb{R}^{N}\right)$, there exists a family $\left(g_{\delta}\right)_{\delta \in(0,1)} \subset L^{p}\left(\mathbb{R}^{N}\right)$ such that $g_{\delta}$ converges to $g$ in $L^{p}\left(\mathbb{R}^{N}\right)$ as $\delta$ goes to zero, and

$$
\varlimsup_{\delta \rightarrow 0} I_{\delta}\left(g_{\delta}\right) \leq I(g) .
$$

Surprisingly, $\left(I_{\delta}\right)$ does not $\Gamma$-converge to $J$ in $L^{p}\left(\mathbb{R}^{N}\right)$ for $p>1$, but it $\Gamma$-converges to $\lambda J$ for some $0<\lambda<1$; the same fact holds for the case $p=1$. More precisely, we have the following.

\section{THEOREM 2}

Let $p \geq 1$, and let $N \geq 1$. Then $\left(I_{\delta}\right) \Gamma$-converges in $L^{p}\left(\mathbb{R}^{N}\right)$ to the functional $I$ defined, for any $g \in L^{p}\left(\mathbb{R}^{N}\right)$, by 


$$
I(g)= \begin{cases}C_{N, p} \int_{\mathbb{R}^{N}}|D g|^{p} d x & \text { if } p>1 \text { and } g \in W^{1, p}\left(\mathbb{R}^{N}\right) \\ & \left(\text { resp., } p=1 \text { and } g \in \mathrm{BV}\left(\mathbb{R}^{N}\right)\right) \\ +\infty & \text { otherwise. }\end{cases}
$$

Here the constant $C_{N, p}$ is defined by (1.3) and satisfies

$$
0<C_{N, p}<\frac{1}{p} K_{N, p} .
$$

For $p \geq 1$ and $N \geq 1$, the definition of the constant $C_{N, p}$ is

$$
C_{N, p}:=\inf \frac{\lim }{\delta \rightarrow 0} \iint_{\left|h_{\delta}(x)-h_{\delta}(y)\right|>\delta} \frac{\delta^{p}}{|x-y|^{N+p}} d x d y,
$$

where the infimum is taken over all families of measurable functions $\left(h_{\delta}\right)_{\delta \in(0,1)}$ defined on the unit open cube $Q$ of $\mathbb{R}^{N}$ such that $h_{\delta}$ converges to $h(x) \equiv\left(x_{1}+\cdots+x_{N}\right) / \sqrt{N}$ in (Lebesgue) measure on $Q$ as $\delta$ goes to zero. We recall here that a family of measurable functions $\left(h_{\delta}\right)_{\delta \in(0,1)}$ defined on a measurable subset $B$ of $\mathbb{R}^{N}$ is said to converge in measure on $B$ to a measurable function $h$ defined on $B$ if and only if, for any $\varepsilon>0$,

$$
\lim _{\delta \rightarrow 0}\left|\left\{x \in B ;\left|h_{\delta}(x)-h(x)\right|>\varepsilon\right\}\right|=0 .
$$

Henceforth, for a measurable subset $A$ of $\mathbb{R}^{N},|A|$ denotes the Lebesgue measure of $A$.

The proof of Theorem 2 is divided into three steps.

Step 1. Proof of property (G2). The main steps of the proof of property (G2) are as follows.

(a) We show that (see Section 2, Lemma 2) there exists a family $\left(h_{\delta}\right)_{\delta \in(0,1)} \subset$ $L^{p}(Q)$ such that $h_{\delta}$ converges to $h(x) \equiv\left(x_{1}+\cdots+x_{N}\right) / \sqrt{N}$ in $L^{p}(Q)$ and (cf. (1.3))

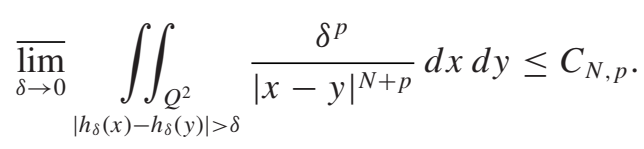

(b) We prove Property (G2) in the case $g$ is "continuous and piecewise linear" with compact support (see Lemma 7) and then obtain property (G2) in the general case by a density argument. The proof of (b) is presented in Section 3. 
Step 2. Proof of property $(G 1)$. Property (G1) is a consequence of the following two propositions.

\section{PROPOSITION 1}

Let $p \geq 1$, let $N \geq 1$, and, let $g \in L^{p}\left(\mathbb{R}^{N}\right)$. Assume that there exists a family $\left(g_{\delta}\right)_{\delta \in(0,1)} \subset L^{p}\left(\mathbb{R}^{N}\right)$ such that $g_{\delta}$ converges to $g$ in $L^{p}\left(\mathbb{R}^{N}\right)$ and

$$
\varliminf_{\delta \rightarrow 0} I_{\delta}\left(g_{\delta}\right)<+\infty
$$

Then $g \in W^{1, p}\left(\mathbb{R}^{N}\right)$ if $p>1$ (resp., $g \in \mathrm{BV}\left(\mathbb{R}^{N}\right)$ if $p=1$ ); moreover,

$$
J(g) \leq C \underline{\lim }_{\delta \rightarrow 0} I_{\delta}\left(g_{\delta}\right)
$$

for some $C>0$ depending only on $N$ and $p$.

Proposition 1 has been proved in [16] (see [16, Theorem 3]); the proof in [16] relies heavily on the ideas of [4].

PROPOSITION 2

Let $p \geq 1$, and let $N \geq 1$. Then for any $g \in W^{1, p}\left(\mathbb{R}^{N}\right)$ if $p>1$ or $g \in \mathrm{BV}\left(\mathbb{R}^{N}\right)$ if $p=1$, and for any family $\left(g_{\delta}\right)_{\delta \in(0,1)} \subset L^{p}\left(\mathbb{R}^{N}\right)$ such that $g_{\delta}$ converges to $g$ in $L^{p}\left(\mathbb{R}^{N}\right)$ as $\delta$ goes to zero, we have

$$
\varliminf_{\delta \rightarrow 0} I_{\delta}\left(g_{\delta}\right) \geq I(g)
$$

The proof of Proposition 2 for the case $p>1$, which is presented in Section 4, follows from the definition of $C_{N, p}$ and the fact that any function in $W^{1, p}(\mathbb{R})$ is locally approximately linear in the sense of measure (see, e.g., [12, Theorem 4, p. 223 ] and the remark below it). When $p=1$, we cannot directly apply the method used in the case $p>1$. In this case, the proof, which is presented in Section 6, relies on Proposition 4, Lemma 14, and a new characterization of BV functions which we introduce in Section 5 (see Proposition 3). This characterization is based on and generalizes the notion of essential variation in the one-dimensional case. It has some advantages in comparison with the classic one (see, e.g., [12, Theorem 2, p. 220], [1, Section 3.11]), which is based on the notion of essential variation on almost every line.

Step 3. Proof of (1.2). Inequality (1.2) is proved in [14].

Concerning the constant $C_{N, p}$, we have the following questions. 
Open question 1

What is the explicit value of $C_{N, p}$ ?

When $N=1$, we made a guess in [14].

Open question 2

Does

$$
C_{1, p}= \begin{cases}\frac{2}{p(p-1)}\left(1-\frac{1}{2^{p-1}}\right) & \text { if } p>1, \\ 2 \ln 2 & \text { if } p=1 ?\end{cases}
$$

The results of this paper were announced in [14].

\section{Preliminaries}

In this section, we prove some useful lemmas which we use later.

LEMMA 1

Let $N \geq 1$, let $p \geq 1$, let $A$ be a measurable subset of $\mathbb{R}^{N}$, and let $f$ and $g$ be two measurable functions defined on A. Define $h_{1}=\min (f, g)$ and $h_{2}=\max (f, g)$. Then

$$
\begin{aligned}
\iint_{\left|h_{1}(x)-h_{1}(y)\right|>\delta} \frac{\delta^{p}}{|x-y|^{N+p}} d x d y \leq & \iint_{|f(x)-f(y)|>\delta} \frac{\delta^{p}}{|x-y|^{N+p}} d x d y \\
& +\iint_{\substack{A^{2} \backslash B_{1}^{2} \\
|g(x)-g(y)|>\delta}} \frac{\delta^{p}}{|x-y|^{N+p}} d x d y
\end{aligned}
$$

and

$$
\begin{aligned}
\iint_{\left|h_{2}(x)-h_{2}(y)\right|>\delta} \frac{\delta^{p}}{|x-y|^{N+p}} d x d y \leq & \iint_{|f(x)-f(y)|>\delta} \frac{\delta^{p}}{|x-y|^{N+p}} d x d y \\
& +\iint_{\substack{A^{2} \backslash B_{2}^{2} \\
|g(x)-g(y)|>\delta}} \frac{\delta^{p}}{|x-y|^{N+p}} d x d y,
\end{aligned}
$$

where

$$
B_{1}=\{x \in A ; f(x) \leq g(x)\} \quad \text { and } \quad B_{2}=\{x \in A ; f(x) \geq g(x)\} .
$$


Moreover, if $g$ is Lipschitz on A with a Lipschitz constant L, then

$$
\iint_{\left|h_{1}(x)-h_{1}(y)\right|>\delta} \frac{\delta^{p}}{|x-y|^{N+p}} d x d y \leq \iint_{|f(x)-f(y)|>\delta} \frac{\delta^{p}}{|x-y|^{N+p}} d x d y+C L^{p}\left|A \backslash B_{1}\right|
$$

and

$$
\iint_{\left|h_{2}(x)-h_{2}(y)\right|>\delta} \frac{\delta^{p}}{|x-y|^{N+p}} d x d y \leq \iint_{|f(x)-f(y)|>\delta} \frac{\delta^{p}}{|x-y|^{N+p}} d x d y+C L^{p}\left|A \backslash B_{2}\right| .
$$

Hereafter in this paper, $C$ denotes a positive constant depending only on $N$ and $p$.

\section{Proof}

It suffices to prove (2.1) and (2.3) since (2.2) and (2.4) follow easily from (2.1) and (2.3).

We first prove (2.1). If $x, y \in B_{1}$, then $\left|h_{1}(x)-h_{1}(y)\right|=|f(x)-f(y)|$. Otherwise $x \notin B_{1}$ or $y \notin B_{1}$. Then $\left|h_{1}(x)-h_{1}(y)\right| \leq \max (|f(x)-f(y)|,|g(x)-g(y)|)$. Hence (2.1) follows.

To obtain (2.3) from (2.1), we remark that

$$
\iint_{\substack{A^{2} \backslash B_{1}^{2} \\|g(x)-g(y)|>\delta}} \frac{\delta^{p}}{|x-y|^{N+p}} d x d y \leq 2 \iiint_{\substack{A \times\left(A \backslash B_{1}\right) \\|g(x)-g(y)|>\delta}} \frac{\delta^{p}}{|x-y|^{N+p}} d x d y
$$

and

$$
\iint_{\substack{A \times\left(A \backslash B_{1}\right) \\|g(x)-g(y)|>\delta}} \frac{\delta^{p}}{|x-y|^{N+p}} d x d y \leq \iint_{\substack{\left(A \backslash B_{1}\right) \times \mathbb{R}^{N} \\|x-y|>\delta / L}} \frac{\delta^{p}}{|x-y|^{N+p}} d x d y \leq C L^{p}\left|A \backslash B_{1}\right|
$$

for any Lipschitz function $g$ on $A$ with a Lipschitz constant $L$.

Here is an obvious consequence of Lemma 1 which we use several times later.

COROLLARY 1

Let $N \geq 1$, let $p \geq 1$, let $-\infty \leq m_{1}<m_{2} \leq+\infty$, let $A$ be a measurable subset of $\mathbb{R}^{N}$, and let $f$ be a measurable function defined on A. Define $h=\min \left(\max \left(f, m_{1}\right), m_{2}\right)$. Then

$$
\iint_{|h(x)-h(y)|>\delta} \frac{\delta^{p}}{|x-y|^{N+p}} d x d y \leq \iint_{|f(x)-f(y)|>\delta} \frac{\delta^{p}}{|x-y|^{N+p}} d x d y .
$$




\section{Remark 1}

Estimate (2.5) was observed and used in [13] and [4].

Another useful and obvious consequence of Lemma 1 is the following.

COROLLARY 2

Let $N \geq 1$, let $p \geq 1$, let $A$ be a measurable subset of $\mathbb{R}^{N}$, let $f$ and $g$ be two measurable functions defined on $A$, and let $c$ be a positive number. Define $h=$ $\min (\max (f, g-c), g+c)$. Suppose that $g$ is Lipschitz on A with a Lipschitz constant L. Then

$$
\iint_{|h(x)-h(y)|>\delta} \frac{\delta^{p}}{|x-y|^{N+p}} d x d y \leq \iint_{|f(x)-f(y)|>\delta} \frac{\delta^{p}}{|x-y|^{N+p}} d x d y+C L^{p}|B|,
$$

where

$$
B:=\{x \in A ;|f(x)-g(x)|>c\} .
$$

An important application of Corollary 2 is the following.

\section{COROLLARY 3}

Let $N \geq 1$, let $p \geq 1$, let $A$ be a measurable subset of $\mathbb{R}^{N}$, let $g$ be a Lipschitz function defined on $A$, let $\left(\delta_{n}\right)_{n \in \mathbb{N}}$ be a sequence of positive numbers converging to zero, and let $\left(g_{n}\right)_{n \in \mathbb{N}}$ be a sequence of measurable functions defined on A such that $g_{n}$ converges to $g$ in measure on $A$. Then there exists a sequence of measurable functions $h_{n}$ defined on $A$ such that $h_{n}$ converges to $g$ uniformly on $A$ and

$$
\varlimsup_{n \rightarrow \infty} \iint_{\left|h_{n}(x)-h_{n}(y)\right|>\delta_{n}} \frac{\delta_{n}^{p}}{|x-y|^{N+p}} d x d y \leq \varlimsup_{n \rightarrow \infty} \iint_{\left|g_{n}(x)-g_{n}(y)\right|>\delta_{n}} \frac{\delta_{n}^{p}}{|x-y|^{N+p}} d x d y .
$$

Proof

Since $g_{n}$ converges to $g$ in measure on $A$, there exists a sequence of positive numbers $\left(c_{n}\right)_{n \in \mathbb{N}}$ converging to zero such that

$$
\lim _{n \rightarrow \infty}\left|A_{n}\right|=0
$$

where

$$
A_{n}:=\left\{x \in A ;\left|g_{n}(x)-g(x)\right|>c_{n}\right\} .
$$

Define $h_{n}=\min \left(\max \left(g_{n}, g-c_{n}\right), g+c_{n}\right)$. Applying Corollary 2, we have

$$
\iint_{A^{2}} \frac{\delta_{n}^{p}}{|x-y|^{N+p}} d x d y \leq \iint_{\left|g_{n}(x)-g_{n}(y)\right|>\delta_{n}} \frac{\delta_{n}^{p}}{|x-y|^{N+p}} d x d y+C L^{p}\left|A_{n}\right|,
$$


where $L$ is a Lipschitz constant of $g$. Therefore,

$$
\varlimsup_{n \rightarrow \infty} \iint_{\left|h_{n}(x)-h_{n}(y)\right|>\delta_{n}} \frac{\delta_{n}^{p}}{|x-y|^{N+p}} d x d y \leq \varlimsup_{n \rightarrow \infty} \iint_{\left|g_{n}(x)-g_{n}(y)\right|>\delta_{n}} \frac{\delta_{n}^{p}}{|x-y|^{N+p}} d x d y .
$$

Using Corollary 3, we can prove the following lemma, which plays an important role in the proof of property $(\mathrm{G} 2)$.

LEMMA 2

Let $N \geq 1$, let $p \geq 1$, let $Q$ be the unit cube of $\mathbb{R}^{N}$, and let $g \equiv\left(\sum_{i=1}^{N} x_{i}\right) / \sqrt{N}$. Then there exist a family of measurable functions $\left(g_{\delta}\right)_{\delta \in(0,1)}$ defined on $Q$ and a family of positive numbers $\left(c_{\delta}\right)_{\delta \in(0,1)}$ converging to zero such that $c_{\delta} \geq \sqrt{\delta},\left|g_{\delta}(x)-g(x)\right| \leq$ $2 N c_{\delta}, g_{\delta}$ is Lipschitz on $Q_{c_{\delta}}$ with a Lipschitz constant 1 , and

$$
\varlimsup_{\delta \rightarrow 0} \iint_{\substack{Q^{2} \\\left|g_{\delta}(x)-g_{\delta}(y)\right|>\delta}} \frac{\delta^{p}}{|x-y|^{N+p}} d x d y \leq C_{N, p}
$$

Hereafter, for $c>0, Q_{c}$ is defined by

$$
Q_{c}:=\left\{x \in Q ; \operatorname{dist}_{\infty}(x, \partial Q) \leq c\right\},
$$

with

$$
\operatorname{dist}_{\infty}(x, A):=\inf _{y \in A} \sup _{i=1, \ldots, N}\left|x_{i}-y_{i}\right|
$$

for any set $A \subset \mathbb{R}^{N}$.

\section{Proof}

It is standard to see that there exist a sequence of positive numbers $\left(\delta_{k}\right)_{k \in \mathbb{N}}$ converging to zero, and a sequence of measurable functions $\left(g_{k}\right)_{k \in \mathbb{N}}$ converging to $g$ in measure on $Q$ such that

$$
\varlimsup_{k \rightarrow \infty} \iint_{\substack{Q^{2} \\\left|g_{k}(x)-g_{k}(y)\right|>\delta_{k}}} \frac{\delta_{k}^{p}}{|x-y|^{N+p}} d x d y \leq C_{N, p} .
$$

Using Corollary 3, one may assume that $g_{k}$ converges to $g$ uniformly on $Q$ as $k$ goes to infinity. Set 


$$
c_{k}=\max \left(\sup _{x \in Q}\left|g_{k}(x)-g(x)\right|, \sqrt{\delta_{k}}\right) .
$$

Define

$$
\left\{\begin{array}{l}
g_{1, k}=\min \left(\max \left(g_{0, k}(x), g\left(0, x_{2}, \ldots, x_{N}\right)+2 c_{k}\right), g\left(1, x_{2}, \ldots, x_{N}\right)-2 c_{k}\right) \\
g_{2, k}=\min \left(\max \left(g_{1, k}(x), g\left(x_{1}, 0, \ldots, x_{N}\right)+4 c_{k}\right), g\left(x_{1}, 1, \ldots, x_{N}\right)-4 c_{k}\right) \\
\ldots \\
g_{N, k}=\min \left(\max \left(g_{N-1, k}(x), g\left(x_{1}, \ldots, x_{N-1}, 0\right)+2 N c_{k}\right), g\left(x_{1}, \ldots, x_{N-1}, 1\right)\right. \\
\left.\quad-2 N c_{k}\right)
\end{array}\right.
$$

with the notation $g_{0, k}=g_{k}$. Then, since

$$
\left\{\begin{array}{l}
g\left(x_{1}, \ldots, x_{i-1}, 0, x_{i+1}, \ldots, x_{N}\right)+2 i c_{k} \leq g(x)+2 i c_{k} \\
g\left(x_{1}, \ldots, x_{i-1}, 1, x_{i+1}, \ldots, x_{N}\right)-2 i c_{k} \geq g(x)-2 i c_{k}
\end{array}\right.
$$

it follows that

$$
g_{i, k}(x) \geq \min \left(g_{i-1, k}(x), g(x)-2 i c_{k}\right)
$$

and

$$
g_{i, k}(x) \leq \max \left(g_{i-1, k}(x), g(x)+2 i c_{k}\right) .
$$

Then, since $g(x)-c_{k} \leq g_{0, k}(x) \leq g(x)+c_{k}$, we have

$$
g(x)-2 i c_{k} \leq g_{i, k}(x) \leq g(x)+2 i c_{k}
$$

for $1 \leq i \leq N$. Since $c_{k}$ is small if $k$ is large, it follows from (2.8) and (2.9) that

$$
g_{i, k}(x)= \begin{cases}g\left(x_{1}, \ldots, x_{i-1}, 0, x_{i+1}, \ldots, x_{N}\right)+2 i c_{k} & \text { if } 0 \leq x_{i} \leq c_{k} \\ g\left(x_{1}, \ldots, x_{i-1}, 1, x_{i+1}, \ldots, x_{N}\right)-2 i c_{k} & \text { if } 1-c_{k} \leq x_{i} \leq 1\end{cases}
$$

for large $k$. Thus from (2.8), $g_{N, k}$ is Lipschitz on $Q_{c_{k}}$ with a Lipschitz constant 1 (which equals $|D g|$ ).

On the other hand, applying Corollary 2, we have

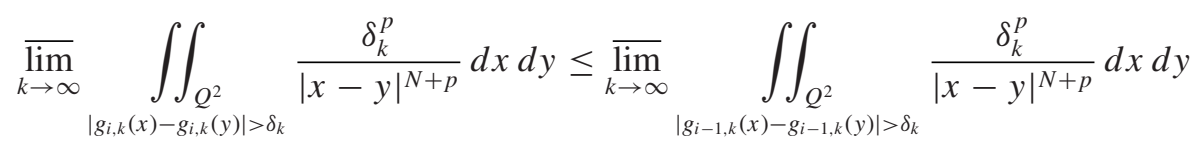

for all $1 \leq i \leq N$, which implies 


$$
\varlimsup_{k \rightarrow \infty} \iint_{g_{N, k}(x)-g_{N, k}(y) \mid>\delta_{k}} \frac{\delta_{k}^{p}}{|x-y|^{N+p}} d x d y \leq \varlimsup_{k \rightarrow \infty} \iint_{\left|g_{k}(x)-g_{k}(y)\right|>\delta_{k}} \frac{\delta_{k}^{p}}{|x-y|^{N+p}} d x d y .
$$

Hence using the arguments above, it suffices to construct a family $\left(h_{\delta}\right)$ such that $\left(h_{\delta}\right)$ converges with $g$ uniformly on $Q$ and

$$
\varlimsup_{\delta \rightarrow 0} \iint_{h_{\delta}(x)-h_{\delta}(y) \mid>\delta} \frac{\delta^{p}}{|x-y|^{N+p}} d x d y \leq C_{N, p} .
$$

For this end, let $\left(\tau_{k}\right)_{k \in \mathbb{N}}$ be a strictly decreasing positive sequence such that $\tau_{k} \leq c_{k} \delta_{k}$. For each $\delta$ small, let $k$ be such that $\tau_{k+1}<\delta \leq \tau_{k}$ ( $k$ is large), and define $m_{1}=\delta_{k} / \delta$ and $m=\left[m_{1}\right]$. Hereafter $[a]$ denotes the largest integer less than $a$.

Define $h_{\delta}^{(1)}:[0, m]^{N} \rightarrow \mathbb{R}$ as follows:

$$
h_{\delta}^{(1)}(y)=\frac{\sum_{i=1}^{N}\left[y_{i}\right]}{\sqrt{N}}+g_{N, k}(x)
$$

where $y=\left(y_{1}, \ldots, y_{N}\right)$ and $x=\left(x_{1}, \ldots, x_{N}\right)$ with $x_{i}=y_{i}-\left[y_{i}\right]$.

For $\alpha \in \mathbb{N}^{N}$ and $c>0$, set

$$
Q_{[\alpha]}:=Q+\left(\alpha_{1}, \ldots, \alpha_{N}\right), \quad Q_{[\alpha], c}:=Q_{c}+\left(\alpha_{1}, \ldots, \alpha_{N}\right)
$$

and

$$
D_{[\alpha], c}:=Q_{[\alpha]} \backslash Q_{[\alpha], c} .
$$

Here $A+\alpha:=\{x+\alpha ; x \in A\}$.

We claim that

$$
\operatorname{Lip}\left(h^{(1)}, B\right) \leq C,
$$

where $B=\bigcup_{\alpha \in\{0, \ldots, m-1\}^{N}}\left(Q_{[\alpha], c_{k}} \backslash Q_{[\alpha], c_{k} / 2}\right)$.

Hereafter $\operatorname{Lip}(f, A)$ denotes the Lipschitz constant of $f$ on $A$ for a function $f$ defined on a subset $A$ of $\mathbb{R}^{N}$. We recall that $C$ denotes a positive constant depending only on $N$ and $p$.

Indeed, since $\operatorname{Lip}\left(g_{N, k}, Q_{c_{k}} \backslash Q_{c_{k} / 2}\right) \leq 1$, it is clear that

$$
\operatorname{Lip}\left(h_{\delta}^{(1)}, Q_{[\alpha], c_{k}} \backslash Q_{[\alpha], c_{k} / 2}\right) \leq 1, \quad \forall \alpha \in\{0, \ldots, m-1\}^{N} .
$$

On the other hand,

$$
\left|h_{\delta}^{(1)}(x)-\frac{\sum_{i=1}^{N} x_{i}}{\sqrt{N}}\right| \leq C c_{k}, \quad \forall x \in[0, m]^{N},
$$


and if $\alpha \neq \alpha^{\prime}$,

$$
|x-y| \geq C c_{k}, \quad \forall x \in Q_{[\alpha], c_{k}} \backslash Q_{[\alpha], c_{k} / 2}, \quad y \in Q_{\left[\alpha^{\prime}\right], c_{k}} \backslash Q_{\left[\alpha^{\prime}\right], c_{k} / 2} .
$$

Combining (2.14), (2.15), and (2.16) yields (2.13).

From (2.13), there exists $h_{\delta}^{(2)}: \mathbb{R}^{N} \rightarrow \mathbb{R}^{N}$ such that $h_{\delta}^{(2)}=h_{\delta}^{(1)}$ on $B$ and

$$
\operatorname{Lip}\left(h_{\delta}^{(2)}, \mathbb{R}^{N}\right) \leq C
$$

Define

$$
h_{\delta}^{(3)}=\left\{\begin{array}{l}
h_{\delta}^{(1)}(x) \text { if } x \in D_{[\alpha], c_{k} / 2} \text { for some } \alpha \in\{0, \ldots, m-1\}^{N}, \\
h_{\delta}^{(2)}(x) \text { otherwise, }
\end{array}\right.
$$

and define

$$
h_{\delta}(x)=\frac{1}{m_{1}} h_{\delta}^{(3)}(m x)
$$

We have

$$
\iint_{\substack{Q^{2} \\\left|h_{\delta}(x)-h_{\delta}(y)\right|>\delta}} \frac{\delta^{p}}{|x-y|^{N+p}} d x d y=\frac{m^{p-N}}{m_{1}^{p}} \underset{\substack{[0, m]^{N} \times[0, m]^{N} \\\left|h_{\delta}^{(3)}(x)-h_{\delta}^{(3)}(y)\right|>\delta_{k}}}{\int} \frac{\delta_{k}^{p}}{|x-y|^{N+p}} d x d y
$$

and

$$
\begin{aligned}
& \quad \iint_{\substack{[0, m]^{N} \times[0, m]^{N} \\
\left|h_{\delta}^{(3)}(x)-h_{\delta}^{(3)}(y)\right|>\delta_{k}}} \frac{\delta_{k}^{p}}{|x-y|^{N+p}} d x d y \\
& \leq \sum_{\substack{\alpha \in \mathbb{N}^{N} \\
1 \leq \alpha_{i} \leq m-1}} \iint_{\substack{D_{[\alpha], c_{k} / 2}^{2} \\
\left|h_{\delta}^{(3)}(x)-h_{\delta}^{(3)}(y)\right|>\delta_{k}}} \frac{\delta_{k}^{p}}{|x-y|^{N+p}} d x d y \\
& +\sum_{\substack{\alpha \in \mathbb{N}^{N} \\
1 \leq \alpha_{i} \leq m-1}} \iint_{\substack{D_{[\alpha], c_{k} / 2} \times\left([0, m]^{N} \backslash D_{\left.[\alpha], c_{k} / 2\right)} \\
\left|h_{\delta}^{(3)}(x)-h_{\delta}^{(3)}(y)\right|>\delta_{k}\right.}} \frac{\delta_{k}^{p}}{|x-y|^{N+p}} d x d y .
\end{aligned}
$$

It is clear from (2.12) and (2.18) that

$$
\iint_{\substack{D_{[\alpha], c_{k} / 2}^{2} \\ h_{\delta}^{(3)}(x)-h_{\delta}^{(3)}(y) \mid>\delta_{k}}} \frac{\delta_{k}^{p}}{|x-y|^{N+p}} d x d y \leq \iint_{\substack{Q^{2} \\\left|g_{N, k}(x)-g_{N, k}(y)\right|>\delta_{k}}} \frac{\delta_{k}^{p}}{|x-y|^{N+p}} d x d y .
$$


From (2.13), (2.17), and (2.18), we have $\operatorname{Lip}\left(h_{\delta}^{(3)},[0, m]^{N} \backslash \bigcup_{\alpha \in\{0, \ldots, m-1\}^{N}} Q_{[\alpha], c_{k}}\right) \leq$ C. This implies, after using (2.7),

$$
\iint_{\substack{D_{[\alpha], c_{k} / 2} \times\left([0, m]^{N} \backslash D_{[\alpha], c_{k} / 2}\right) \\\left|h_{\delta}^{(3)}(x)-h_{\delta}^{(3)}(y)\right|>\delta_{k}}} \frac{\delta_{k}^{p}}{|x-y|^{N+p}} d x d y \leq C\left(c_{k}+\delta_{k}^{p} / c_{k}^{p}\right) \leq C\left(c_{k}+\delta_{k}^{p / 2}\right) .
$$

Combining (2.20), (2.21), and (2.22) yields

$$
\begin{aligned}
& \iint_{[0, m]^{N}} \frac{\delta_{k}^{p}}{|x-y|^{N+p}} d x d y \\
& \leq m^{N} \iint_{\substack{\mid 0,1]^{N} \\
g_{N, k}^{(3)}(x)-h_{\delta}^{(3)}(y) \mid>\delta_{k}}} \frac{\delta_{k}^{p}}{|x-y|^{N+p}} d x d y+\operatorname{Cm}^{N}\left(c_{k}+\delta_{k}^{\frac{p}{2}}\right)
\end{aligned}
$$

Since $m \leq m_{1}$, we deduce from (2.19) and (2.23) that

$$
\iint_{Q^{2}} \frac{\delta^{p}}{|x-y|^{N+p}} d x d y \leq \iint_{\left|h_{N, k}(x)-h_{\delta}(y)\right|>\delta} \frac{\delta_{k, k}^{p}}{|x-y|^{N+p}} d x d y+C\left(c_{k}+\delta_{k}^{p / 2}\right) .
$$

Therefore, it follows from (2.11), (2.6), and (2.24) that

$$
\varlimsup_{\delta \rightarrow 0} \iint_{h_{\delta}(x)-h_{\delta}(y) \mid>\delta} \frac{\delta^{p}}{|x-y|^{N+p}} d x d y \leq C_{N, p}
$$

since $\lim _{k \rightarrow \infty} \delta_{k}=\lim _{k \rightarrow \infty} c_{k}=0$.

From (2.18), it is clear that $h_{\delta}$ converges to $g$ uniformly on $Q$.

\section{Proof of property (G2)}

The proof of property (G2) is derived after establishing several lemmas. The first one, which is used in the proof of Lemma 4, deals with a covering result which is quite classic for experts; however, we cannot find any reference for it. For the convenience of the reader, the proof is presented.

\section{LEMMA 3}

Let $\Omega$ be a nonempty open subset of $\mathbb{R}^{N}$, and let $B$ be a nonempty bounded open subset of $\mathbb{R}^{N}$ with $|\partial B|=0$. Then there exists a collection of open subsets $\left(B_{i}\right)_{i \in \mathbb{N}}$ such that $B_{i} \subset \Omega, B_{i}$ is an image of $B$ by a dilatation and a translation, $B_{i} \cap B_{j}=\varnothing$ for $i \neq j$, and $\sum_{i \in \mathbb{N}}\left|B_{i}\right|=|\Omega|$. 


\section{Proof}

Let $\tilde{B}$ be an image of $B$ by a dilatation and a translation such that the closure of $\tilde{B}$ is included in $Q$. Since $B$ is open, $|\tilde{B}|=c>0$. Set $\Omega_{0}=\Omega$. Consider a collection $\left(Q_{1, i}\right)_{i \in \mathbb{N}}$ such that $Q_{1, i} \subset \Omega_{0}, Q_{1, i}$ is an image of $Q$ by a dilatation and a translation, $Q_{1, i} \cap Q_{1, j}=\varnothing$ for $i \neq j$, and $|\Omega|=\sum_{i \in \mathbb{N}}\left|Q_{1, i}\right|$. The existence of this collection follows from [19, assertion $(d)$, p. 50]. Then there exists a collection of disjoint sets $\left(B_{1, i}\right)_{i \in \mathbb{N}}$ such that $B_{1, i}$ is an image of $B$ by a translation and a dilatation, $\bar{B}_{1, i} \subset Q_{1, i}$, and $\left|B_{1, i}\right|=c\left|Q_{1, i}\right|$. This implies that $\sum_{i \in \mathbb{N}}\left|B_{1, i}\right|=c\left|\Omega_{0}\right|$. Set $\Omega_{1}=\bigcup_{i \in \mathbb{N}}\left(Q_{1, i} \backslash \bar{B}_{1, i}\right)$. Then $\Omega_{1}$ is open and $\left|\Omega_{1}\right|=(1-c)\left|\Omega_{0}\right|($ since $|\partial B|=0)$. Continuing this process, we find collections of sets $\left(Q_{k, i}\right)_{(k, i) \in \mathbb{N}^{2}}$ and $\left(B_{k, i}\right)_{(k, i) \in \mathbb{N}^{2}}$, and open subsets $\left(\Omega_{k}\right)_{k \in \mathbb{N}}$ of $\mathbb{R}^{N}$ such that $Q_{k, i}$ and $B_{k, i}$ are images of $Q$ and $B$, respectively, by a dilatation and a translation, $Q_{k, i} \subset \Omega_{k-1}, Q_{k, i} \cap Q_{k, j}=\varnothing$ for $i \neq j$, $\sum_{i \in \mathbb{N}}\left|Q_{k, i}\right|=\left|\Omega_{k-1}\right|, \bar{B}_{k, i} \subset Q_{k, i},\left|B_{k, i}\right|=c\left|Q_{k, i}\right|$, and $\Omega_{k}=\bigcup_{i \in \mathbb{N}}\left(Q_{k, i} \backslash \bar{B}_{k, i}\right)$. Set

$$
a_{m}=\sum_{k=1}^{m} \sum_{i \in \mathbb{N}}\left|B_{k, i}\right|
$$

Then since

$$
\sum_{k=1}^{m} \sum_{i \in \mathbb{N}}\left|B_{k, i}\right|=c\left(|\Omega|-\sum_{k=1}^{m-1} \sum_{i \in \mathbb{N}}\left|B_{k, i}\right|\right)+\sum_{k=1}^{m-1} \sum_{i \in \mathbb{N}}\left|B_{k, i}\right|,
$$

we have

$$
a_{m}=c\left(|\Omega|-a_{m-1}\right)+a_{m-1} .
$$

It is easy to see that $a_{m}$ is increasing and bounded from above. Hence $a_{m}$ converges to $a$. Thus from (3.1), $a=|\Omega|$. The conclusion follows by taking the collection $\left(B_{i, k}\right)_{(i, k) \in \mathbb{N}^{2}}$.

LEMMA 4

Let $S$ be an open subset of $\mathbb{R}^{N}$ with $|\partial S|=0$, and let $g$ be an affine function defined on S. Then

$$
\inf \frac{\lim _{\delta \rightarrow 0}}{\int_{\substack{S \times S \\\left|g_{\delta}(x)-g_{\delta}(y)\right|>\delta}}} \frac{\delta^{p}}{|x-y|^{N+p}} d x d y=C_{N, p}|D g|^{p}|S|,
$$

where the infimum is taken over all families of measurable functions $\left(g_{\delta}\right)_{\delta \in(0,1)}$ defined on $S$ such that $g_{\delta}$ converges to $g$ in measure on $S$ as $\delta$ goes to zero. Moreover, there exists a family of measurable functions $\left(h_{\delta}\right)_{\delta \in(0,1)}$ such that $h_{\delta}$ converges to $g$ uniformly 
on $S$ and

$$
\varlimsup_{\delta \rightarrow 0} \iint_{\substack{S \times S \\\left|h_{\delta}(x)-h_{\delta}(y)\right|>\delta}} \frac{\delta^{p}}{|x-y|^{N+p}} d x d y=C_{N, p}|D g|^{p}|S| .
$$

Proof

After using a rotation, a dilatation, and a translation, one may assume that $g=$ $\left(\sum_{i=1}^{N} x_{i}\right) / \sqrt{N}$ :

Set

$$
\tilde{C}_{N, p}=\inf \frac{\lim }{\delta \rightarrow 0} \iint_{\left|g_{\delta}(x)-g_{\delta}(y)\right|>\delta} \frac{\delta^{p}}{|x-y|^{N+p}} d x d y,
$$

where the infimum is taken over all families of measurable functions $\left(g_{\delta}\right)_{\delta \in(0,1)}$ defined on $S$ such that $g_{\delta}$ converges to $g$ in measure as $\delta$ goes to zero.

\section{Claim 1}

We have $\tilde{C}_{N, p} \geq C_{N, p}|S|$.

\section{Claim 2}

We have $\tilde{C}_{N, p} \leq C_{N, p}|S|$, and there exists a family of measurable functions $\left(h_{\delta}\right)_{\delta \in(0,1)}$ defined on $S$ such that $h_{\delta}$ converges to $g$ uniformly on $S$, and

$$
\varlimsup_{\delta \rightarrow 0} \iint_{\left|h_{\delta}(x)-h_{\delta}(y)\right|>\delta} \frac{\delta^{p}}{|x-y|^{N+p}} d x d y=C_{N, p}|S| .
$$

It is clear that the conclusion follows from Claims 1 and 2.

Proof of Claim 1. Let $\left(g_{\delta}\right)_{\delta \in(0,1)}$ be a family of measurable functions which converges to $g$ in measure on $S$. By Lemma 3, there exists a sequence of sets $\left(Q_{i}\right)_{i \in \mathbb{N}}$ such that $Q_{i}$ is an image of $Q$ by a dilatation and a translation, $Q_{i} \cap Q_{j}=\varnothing$ for $i \neq j, Q_{i} \subset S$, and

$$
|S|=\sum_{i \in \mathbb{N}}\left|Q_{i}\right|
$$

From the definition of $C_{N, p}$, by a change of variables, we have

$$
\varliminf_{\delta \rightarrow 0} \iint_{\substack{Q_{i}^{2} \\\left|g_{\delta}(x)-g_{\delta}(y)\right|>\delta}} \frac{\delta^{p}}{|x-y|^{N+p}} d x d y \geq C_{N, p}\left|Q_{i}\right|,
$$


which implies

$$
\varliminf_{\delta \rightarrow 0} \iint_{\left|g_{\delta}(x)-g_{\delta}(y)\right|>\delta} \frac{\delta^{p}}{|x-y|^{N+p}} d x d y \geq C_{N, p} \sum_{i \in \mathbb{N}}\left|Q_{i}\right|=C_{N, p}|S| .
$$

Claim 1 now follows from (3.2) and (3.3).

Proof of Claim 2. We prove Claim 2 by contradiction. Suppose that this is not true. Then there exists $\varepsilon_{0}>0$ such that

$$
\varlimsup_{\delta \rightarrow 0} \iint_{\left|h_{\delta}(x)-h_{\delta}(y)\right|>\delta} \frac{\delta^{p}}{|x-y|^{N+p}} d x d y \geq\left(C_{N, p}+\varepsilon_{0}\right)|S|
$$

for any family of measurable functions $\left(h_{\delta}\right)_{\delta \in(0,1)}$ such that $h_{\delta}$ uniformly converges to $g$ on $S$. Let $\left(g_{\delta}\right)_{\delta \in(0,1)}$ be a family of measurable functions defined on $Q$ such that $g_{\delta}$ converges to $g$ uniformly on $Q$ and

$$
\varlimsup_{\delta \rightarrow 0} \iint_{Q_{\delta}(x)-g_{\delta}(y) \mid>\delta} \frac{\delta^{p}}{|x-y|^{N+p}} d x d y=C_{N, p}
$$

The existence of $\left(g_{\delta}\right)_{\delta \in(0,1)}$ is affirmed by Lemma 2 . From Lemma 3, there exists a collection of sets $\left(S_{i}\right)_{i \in \mathbb{N}}$ such that $S_{i}$ is an image of $S$ by a dilatation and a translation for $i \in \mathbb{N}, S_{i} \cap S_{j}=\varnothing$ for $i \neq j, S_{i} \subset Q$, and

$$
|Q|=\sum_{i \in \mathbb{N}}\left|S_{i}\right|
$$

Then, by a change of variables, it follows from (3.4) that

$$
\varlimsup_{\delta \rightarrow 0} \iint_{\substack{S_{i}^{2} \\\left|g_{\delta}(x)-g_{\delta}(y)\right|>\delta}} \frac{\delta^{p}}{|x-y|^{N+p}} d x d y \geq\left(C_{N, p}+\varepsilon_{0}\right)\left|S_{i}\right|
$$

This implies

$$
\varlimsup_{\delta \rightarrow 0} \iint_{\substack{g_{\delta}(x)-g_{\delta}(y) \mid>\delta \\ \lim ^{2}}} \frac{\delta^{p}}{|x-y|^{N+p}} d x d y \geq\left(C_{N, p}+\varepsilon_{0}\right) \sum_{i \in \mathbb{N}}\left|S_{i}\right|=\left(C_{N, p}+\varepsilon_{0}\right)|Q| .
$$

This contradicts the choice of $\left(g_{\delta}\right)$.

We next introduce the following notation. 


\section{Definition 1}

Let $A_{1}, A_{2}, \ldots, A_{m}$ be disjoint open $(N+1)$-simplices in $\mathbb{R}^{N}$ such that every coordinate component of any vertex of $A_{i}$ is equal to zero or $1, A_{i} \cap A_{j}=\varnothing$ for $i \neq j$

$$
\bar{Q}=\bigcup_{i=1}^{m} \bar{A}_{i}
$$

and

$$
A_{1}=\left\{x=\left(x_{1}, \ldots, x_{N}\right) \in \mathbb{R}^{N} ; x_{i}>0 \text { for all } 1 \leq i \leq N, \text { and } \sum_{i=1}^{N} x_{i}<1\right\} .
$$

The following lemma is a variant of Lemma 2 for $\left\{A_{\ell}\right\}_{\ell=1}^{m}$.

\section{LEMMA 5}

Let $\ell \in\{1, \ldots, m\}$, and let $g$ be an affine function defined on $A_{\ell}$ such that $\frac{\partial g}{\partial n} \neq 0$ along the boundary of $A_{\ell}$. Then there exists a family of measurable functions $\left(g_{\delta}\right)_{\delta \in(0,1)}$ defined on $A_{i}$ and a family of positive numbers $\left(c_{\delta}\right)_{\delta \in(0,1)}$ converging to zero such that $c_{\delta} \geq \sqrt{\delta},\left|g_{\delta}(x)-g(x)\right| \leq 8 N(|D g|+1) c_{\delta}$ for all $x \in A_{\ell}, g_{\delta}$ is Lipschitz on $A_{\ell, c_{\delta}}$, where

$$
A_{\ell, c_{\delta}}:=\left\{x \in A_{\ell} ; \operatorname{dist}_{\infty}\left(x, A_{\ell}^{c}\right) \leq c_{\delta}\right\}
$$

with a Lipschitz constant $|D g|$, and

$$
\varlimsup_{\delta \rightarrow 0} \underset{\substack{A_{\ell} \times A_{\ell} \\\left|g_{\delta}(x)-g_{\delta}(y)\right|>\delta}}{\int} \frac{\delta^{p}}{|x-y|^{N+p}} d x d y \leq C_{N, p}|D g|^{p}\left|A_{\ell}\right| .
$$

\section{Proof}

It suffices to prove the case $\ell=1$. We adapt here the idea used in the proof of Lemma 2. By Lemma 4, there exists a family of measurable functions $\left(g_{\delta}\right)_{\delta \in(0,1)}$ such that $g_{\delta}$ converges to $g$ uniformly on $A_{1}$ as $\delta$ goes to zero, and

$$
\varlimsup_{\delta \rightarrow 0} \iint_{\substack{A_{1} \times A_{1} \\\left|g_{\delta}(x)-g_{\delta}(y)\right|>\delta}} \frac{\delta^{p}}{|x-y|^{N+p}} d x d y=C_{N, p}|D g|^{p}\left|A_{1}\right| .
$$


Set

$$
c_{\delta}=\max \left(\left\|g_{\delta}-g\right\|_{L^{\infty}\left(A_{1}\right)}, \sqrt{\delta}\right), \quad l_{\delta}=2\left(|D g| c_{\delta}+c_{\delta}\right),
$$

and

$$
g_{0, \delta}=g_{\delta},
$$

for $\delta \in(0,1)$. For $i=1,2, \ldots, N$, define

$g_{i, \delta}(x)= \begin{cases}\max \left(g_{i-1, \delta}(x), g\left(x_{1}, \ldots, x_{i-1}, 0, x_{i+1}, \ldots, x_{N}\right)+i l_{\delta}\right) & \text { if } \frac{\partial g}{\partial x_{i}}>0, \\ \min \left(g_{i-1, \delta}(x), g\left(x_{1}, \ldots, x_{i-1}, 0, x_{i+1}, \ldots, x_{N}\right)-i l_{\delta}\right) & \text { if } \frac{\partial g}{\partial x_{i}}<0 .\end{cases}$

Set $e=(1 / \sqrt{N}, \ldots, 1 / \sqrt{N})$, and define

$$
g_{N+1, \delta}(x)= \begin{cases}\max \left(g_{N, \delta}(x), g(z(x))+(N+1) l_{\delta}\right) & \text { if } \frac{\partial g}{\partial e}<0 \\ \min \left(g_{N, \delta}(x), g(z(x))-(N+1) l_{\delta}\right) & \text { if } \frac{\partial g}{\partial e}>0\end{cases}
$$

Here for each $x \in \mathbb{R}^{N}, z(x)=x-\langle x, e\rangle e+e$; that is, $z(x)$ denotes the projection of $x$ on the hyperplane $P$ which is orthogonal to $e$ and contains $e$. In the same way, to obtain (2.10) in the proof of Lemma 1, we have, for $\delta$ small (this is assumed in what follows),

$$
\left|g_{i, \delta}(x)-g(x)\right| \leq i l_{\delta}, \quad \forall x \in A_{1}
$$

This implies, by (3.5) and (3.6),

$$
g_{i, \delta}(x)=\left\{\begin{array}{l}
g\left(x_{1}, \ldots, x_{i-1}, 0, x_{i+1}, \ldots, x_{N}\right)+i l_{\delta} \text { if } \frac{\partial g}{\partial x_{i}}>0 \\
g\left(x_{1}, \ldots, x_{i-1}, 0, x_{i+1}, \ldots, x_{N}\right)-i l_{\delta} \text { if } \frac{\partial g}{\partial x_{i}}<0
\end{array}\right.
$$

for $1 \leq i \leq N$, for any $x \in A_{1}$ such that $0 \leq x_{i} \leq c_{\delta}$, and

$$
g_{N+1, \delta}(x)=\left\{\begin{array}{l}
g(z(x))+(N+1) l_{\delta} \text { if } \frac{\partial g}{\partial e}<0 \\
g(z(x))-(N+1) l_{\delta} \text { if } \frac{\partial g}{\partial e}>0,
\end{array}\right.
$$

for any $x \in A_{1}$ such that $0 \leq|x-z(x)| \leq c_{\delta}$, where $z(x)=x-\langle x, e\rangle e+e$. Then $g_{N+1, \delta}$ is Lipschitz on $A_{1, c_{\delta}}$ with a Lipschitz constant $|D g|$. 
It remains to prove

$$
\varlimsup_{\delta \rightarrow 0} \iint_{\substack{A_{1} \times A_{1} \\\left|g_{N+1, \delta}(x)-g_{N+1, \delta}(y)\right|>\delta}} \frac{\delta^{p}}{|x-y|^{N+p}} d x d y \leq \varlimsup_{\delta \rightarrow 0} \iint_{\substack{A_{1} \times A_{1} \\\left|g_{\delta}(x)-g_{\delta}(y)\right|>\delta}} \frac{\delta^{p}}{|x-y|^{N+p}} d x d y .
$$

Since $\frac{\partial g}{\partial n} \neq 0$, it follows from (3.5) and (3.6) that $g_{i, \delta}$ converges to $g$ in measure in $A_{1}$. Applying Lemma 2, we have, for $1 \leq i \leq N+1$,

$$
\varlimsup_{\delta \rightarrow 0} \iint_{\substack{A_{1} \times A_{1} \\\left|g_{i, \delta}(x)-g_{i, \delta}(y)\right|>\delta}} \frac{\delta^{p}}{|x-y|^{N+p}} d x d y \leq \varlimsup_{\delta \rightarrow 0} \iint_{\substack{A_{1} \times A_{1} \\\left|g_{i-1, \delta}(x)-g_{i-1, \delta}(y)\right|>\delta}} \frac{\delta^{p}}{|x-y|^{N+p}} d x d y,
$$

which implies (3.7).

To approximate a smooth function by a family of continuous piecewise linear functions and to be able to apply Lemma 5, we introduce the following.

\section{Definition 2}

For each $k \in \mathbb{N}, K$ is called a $k$-net of $\mathbb{R}^{N}$ if and only if there exist $z \in \mathbb{Z}^{N}$ and $\ell \in\{1,2, \ldots, m\}$ such that $K=\left(1 / 2^{k}\right) A_{\ell}+z / 2^{k}$.

Hereafter, for any two subsets $A$ and $B$ of $\mathbb{R}^{N}$ and a real number $c$, we define

$$
c A=\left\{c a \in \mathbb{R}^{N} ; a \in A\right\}
$$

and

$$
A+B:=\left\{a+b \in \mathbb{R}^{N} ; a \in A \text { and } b \in B\right\} .
$$

When $B$ is a set containing only a vector $v$, we write $A+v$ instead of $A+\{v\}$.

\section{Definition 3}

Function $g$ on $\mathbb{R}^{N}$ is said to be a continuous piecewise linear function defined on $k$-nets if and only if $g$ is affine on each $k$-net of $\mathbb{R}^{N}$.

The following result follows immediately from Lemma 5 after a change of variables.

LEMMA 6

Let $K$ be a $k$-net of $\mathbb{R}^{N}$, and let $g$ be an affine function defined on $K$ such that $\frac{\partial g}{\partial n} \neq 0$ along the boundary of $K$. Then there exists a family of measurable functions $\left(g_{\delta}\right)_{\delta \in(0,1)}$ defined on $K$ and a family of positive numbers $\left(c_{\delta}\right)_{\delta \in(0,1)}$ converging to zero such that 
$c_{\delta} \geq \sqrt{\delta},\left|g_{\delta}(x)-g(x)\right| \leq 2^{-k+3} N(|D g|+1) c_{\delta}$ for all $x \in K, g_{\delta}$ is a Lipschitz function on $K_{2^{-k} c_{\delta}}$ with a Lipschitz constant $|D g|$, and

$$
\varlimsup_{\delta \rightarrow 0} \iint_{\substack{K \times K \\\left|g_{\delta}(x)-g_{\delta}(y)\right|>\delta}} \frac{\delta^{p}}{|x-y|^{N+p}} d x d y \leq C_{N, p}|D g|^{p}|K| .
$$

Hereafter

$$
K_{\tau}:=\left\{x \in K ; \operatorname{dist}_{\infty}\left(x, K^{c}\right) \leq \tau\right\}, \quad \forall \tau>0 .
$$

We are now ready to prove property (G2) for a "continuous piecewise linear function" with compact support.

\section{LEMMA 7}

Let $g$ be a continuous piecewise linear function on $k$-nets with compact support such that on each $k$-net, $\frac{\partial g}{\partial n} \neq 0$ along the boundary of that $k$-net unless $g$ is constant on this one. Then there exists a family of measurable functions $\left(g_{\delta}\right)_{\delta \in(0,1)} \subset L^{p}\left(\mathbb{R}^{N}\right)$ such that $g_{\delta}$ converges to $g$ in $L^{p}\left(\mathbb{R}^{N}\right) \cap L^{\infty}\left(\mathbb{R}^{N}\right)$, and

$$
\varlimsup_{\delta \rightarrow 0} \iint_{\substack{\mathbb{R}^{N} \times \mathbb{R}^{N} \\\left|g_{\delta}(x)-g_{\delta}(y)\right|>\delta}} \frac{\delta^{p}}{|x-y|^{N+p}} d x d y \leq C_{N, p} \int_{\mathbb{R}^{N}}|D g|^{p} d x .
$$

Proof

For each $k$-net $K$, if $g$ is not constant on $K$, by Lemma 6 there exist a family of measurable functions $\left(h_{i, \delta}\right)_{\delta \in(0,1)}$ defined on $K$ and a family of positive numbers $\left(c_{K, \delta}\right)_{\delta \in(0,1)}$ converging to zero such that $c_{K, \delta} \geq \sqrt{\delta},\left|h_{K \delta}(x)-g(x)\right| \leq 2^{-k+3} N\left(\|D g\|_{\infty}+1\right) c_{K, \delta}$ for $x \in K, h_{K, \delta}$ is Lipschitz on $K_{2^{-k} c_{K, \delta}}$ with a Lipschitz constant $\|D g\|_{L^{\infty}\left(\mathbb{R}^{N}\right)}$, and

$$
\varlimsup_{\delta \rightarrow 0} \iint_{h_{K, \delta}(x)-h_{K, \delta}(y) \mid>\delta} \frac{\delta^{p}}{|x-y|^{N+p}} d x d y \leq C_{N, p} \int_{K}|D g|^{p} d x .
$$

If $g$ is constant on $K$, define $h_{K, \delta}=g$ on $K, c_{K, \delta}=\sqrt{\delta}$.

We now follow the ideas used in the proof of Lemma 2. Define $g_{\delta}^{(1)}: \mathbb{R}^{N} \rightarrow \mathbb{R}$ by $g_{\delta}(x)=h_{K, \delta}(x)$ if $x \in K$. In the same way, to construct $h_{\delta}^{(3)}$ from $h_{\delta}^{(1)}$ in the proof of Lemma 2, from $g_{\delta}^{(1)}$ we can construct $g_{\delta}$ such that $g_{\delta}(x)=g_{\delta}^{(1)}(x)$ if $x \in K_{2^{-k-1} c_{K, \delta}}$ and $g_{\delta}$ is Lipschitz on $\mathbb{R}^{N} \backslash \bigcup_{K} \bar{K}_{2^{-k} c_{K, \delta}}$ with a Lipschitz constant $C\left(\|D g\|_{\infty}+1\right)$. Moreover, one can assume that $\operatorname{supp} g_{\delta} \subset \operatorname{supp} g+B_{1}$ (the unit ball of $\mathbb{R}^{N}$ ) as $\delta$ is 
small. Hence similarly to (2.23), we have

$$
\begin{aligned}
& \iint_{\substack{\mathbb{R}^{N} \times \mathbb{R}^{N} \\
\delta_{\delta}(x)-g_{\delta}(y) \mid>\delta}} \frac{\delta^{p}}{|x-y|^{N+p}} d x d y \\
& \leq \sum_{K} \int_{\left|h_{K, \delta}(x)-h_{K, \delta}(y)\right|>\delta} \frac{\delta^{p}}{|x-y|^{N+p}} d x d y+C(k, g)\left(\max _{K} c_{K, \delta}+\delta^{\frac{p}{2}}\right) .
\end{aligned}
$$

This implies

$$
\varlimsup_{\delta \rightarrow 0} \underset{\substack{\mathbb{R}^{N} \times \mathbb{R}^{N} \\\left|g_{\delta}(x)-g_{\delta}(y)\right|>\delta}}{\int} \frac{\delta^{p}}{|x-y|^{N+p}} d x d y \leq C_{N, p} \int_{\mathbb{R}^{N}}|D g|^{p} d x .
$$

Proof of property (G2)

Let $\left(g_{n}\right)_{n \in \mathbb{N}}$ be a sequence of smooth functions with compact support in $\mathbb{R}^{N}$ such that $g_{n}$ converges to $g$ in $L^{p}\left(\mathbb{R}^{N}\right)$ and $\left\|D g_{n}\right\|_{L^{p}\left(\mathbb{R}^{N}\right)}$ converges to $\|D g\|_{L^{p}\left(\mathbb{R}^{N}\right)}$. (When $p=1$, the $L^{1}$-norm is replaced by the total mass.) For each $n \in \mathbb{N}$, let $\left(g_{k, n}\right)_{k \in \mathbb{N}}$ be a sequence of functions defined on $\mathbb{R}^{N}$ such that $g_{k, n}$ is a continuous piecewise linear function with compact support defined on $k$-nets and $g_{k, n}$ converges to $g_{n}$ in $W^{1, p}\left(\mathbb{R}^{N}\right)$. Without loss of generality, one may assume that $\frac{\partial g_{k, n}}{\partial n} \neq 0$ along the boundary of each $k$-net unless $g_{k, n}$ is constant on this one. Applying Lemma 7, we find a family $\left(g_{\delta, k, n}\right)_{\delta \in(0,1)} \subset L^{p}\left(\mathbb{R}^{N}\right)$ such that $g_{\delta, k, n}$ converges to $g_{k, n}$ in $L^{p}\left(\mathbb{R}^{N}\right)$, as $\delta$ goes to zero, and

$$
\varlimsup_{\delta \rightarrow 0} I_{\delta}\left(g_{\delta, k, n}\right) \leq C_{N, p} \int_{\mathbb{R}^{N}}\left|D g_{k, n}\right|^{p} d x .
$$

The rest of the proof, which is quite standard, is left to the reader.

\section{Proof of Proposition 2 in the case $p>1$}

We begin this section with the following result, which is a consequence of Lemma 4.

\section{LEMMA 8}

Let $p \geq 1$, let $\varepsilon>0$, and let $\tilde{Q}$ be an image of $Q$, the unit cube of $\mathbb{R}^{N}$, by a translation and a dilatation. Let $l$ be the edge length of $\tilde{Q}$. Then there exist three positive numbers $\delta_{1}, \delta_{2}$, and $\delta_{3}$ depending only on $\varepsilon$ such that if $g$ is a measurable function defined on $\tilde{Q}$,

$$
\left|\left\{x \in \tilde{Q} ;|g(x)-(\langle a, x\rangle+b)|>l|a| \delta_{1}\right\}\right|<\delta_{2}|\tilde{Q}|
$$


and $\delta<l|a| \delta_{3}$, for some $a \in \mathbb{R}^{N}$ and $b \in \mathbb{R}$, then

$$
\iint_{\tilde{Q}^{2}} \frac{\delta^{p}}{|x-y|^{N+p}} d x d y \geq\left(C_{N, p}-\varepsilon\right)|a|^{p}|\tilde{Q}| .
$$

Hereafter $\langle\cdot, \cdot\rangle$ denotes the usual scalar product in $\mathbb{R}^{N}$.

\section{Proof}

By a change of variables, without loss of generality, it suffices to prove Lemma 8 in the case $\tilde{Q}=Q$ and $|a|=1$. We prove this by contradiction. Suppose that this is not true. Then there exist $\varepsilon_{0}>0$, a sequence of measurable functions $\left(g_{n}\right)_{n \in \mathbb{N}}$, a sequence $\left(a_{n}\right)_{n \in \mathbb{N}} \subset \mathbb{R}^{N}$, a sequence $\left(b_{n}\right)_{n \in \mathbb{N}} \subset \mathbb{R}$, and a sequence $\left(\delta_{n}\right)_{n \in \mathbb{N}}$ converging to zero such that $\left|a_{n}\right|=1$,

$$
\left|\left\{x \in Q ;\left|g_{n}(x)-\left(\left\langle a_{n}, x\right\rangle+b_{n}\right)\right|>\frac{1}{n}\right\}\right|<\frac{1}{n},
$$

and

$$
\iint_{Q_{Q^{2}}(x)-g_{n}(y) \mid>\delta_{n}} \frac{\delta_{n}^{p}}{|x-y|^{N+p}} d x d y<C_{N, p}-\varepsilon_{0} .
$$

Without loss of generality, one may assume that $b_{n}=0$ for $n \in \mathbb{N}$. Since $\left|a_{n}\right|=1$, there exist $a \in \mathbb{R}^{N}$ and a subsequence $\left(a_{n_{k}}\right)$ of $\left(a_{n}\right)$ such that $a_{n_{k}}$ converges to $a$ and $|a|=1$. Then $g_{n_{k}}$ converges to $\langle a, \cdot\rangle$ in measure on $Q$ and

$$
\iint_{Q^{2}} \frac{\delta_{n_{k}}^{p}}{|x-y|^{N+p}} d x d y<C_{N, p}-\varepsilon_{0} .
$$

This contradicts Lemma 4.

We are ready to give the following.

Proof of Proposition 2 in the case $p>1$

In the proof we essentially use the following result (see, e.g., [12, Theorem 1, p. 228]). Let $f \in W_{l o c}^{1, p}\left(\mathbb{R}^{N}\right)$. Then for almost every $x \in \mathbb{R}^{N}$,

$$
\lim _{r \rightarrow 0} \frac{1}{r} f_{B(x, r)}|f(y)-f(x)-\langle D f(x), y-x\rangle| d y=0 .
$$

Here $B(x, r)$ denotes the ball of $\mathbb{R}^{N}$ centered at $x$ with radius $r$. 
For $x \in \mathbb{R}^{N}$ and $r>0$, let $Q(x, r)$ denote the open cube centered at $x$ with edge length $2 r$; that is,

$$
Q(x, r):=\left\{y=\left(y_{1}, \ldots, y_{N}\right) \in \mathbb{R}^{N} ;\left|y_{i}-x_{i}\right|<r \quad \text { for all } 1 \leq i \leq N\right\} .
$$

For $n=1,2,3, \ldots$, set $P_{n}=2^{-n} \mathbb{Z}^{N}$, and let $\Omega_{n}$ be the collection of all open cubes with edge length $2^{-n}$ whose corners belong to $P_{n}$. For $x \in \mathbb{R}^{N}$, define

$$
\rho_{n}(x):=\sup _{0<r<2^{-n+1}} \frac{1}{r} f_{Q(x, r)}|g(y)-g(x)-\langle D g(x), y-x\rangle| d y
$$

and

$$
\tau_{n}(x):=\sup _{\substack{Q^{\prime} \in \Omega_{k} ; k \geq n ; \\ Q^{\prime} \text { contains } x}} f_{Q^{\prime}}|D g(y)-D g(x)|^{p} d y
$$

and for $m \in \mathbb{N}$, set

$$
A_{m}=\left\{\begin{array}{l}
x \in[-m, m]^{N} \\
\frac{1}{m} \leq|D g(x)| \leq m, \lim _{n \rightarrow \infty} \rho_{n}(x)=0, \text { and } \lim _{n \rightarrow \infty} \tau_{n}(x)=0
\end{array}\right\} .
$$

Fix $\varepsilon>0$ (arbitrary), and let $\delta_{1}, \delta_{2}$, and $\delta_{3}$ be three positive constants corresponding to $\varepsilon$ in Lemma 8. By (4.1), the theory of maximal functions, and the Egorov theorem, it follows from (4.4) that there exist $m \in \mathbb{N}_{+}$and a compact set $B_{m} \subset A_{m}$ such that $\rho_{n}$ and $\tau_{n}$ converge to zero uniformly on $B_{m}$, and

$$
\int_{\mathbb{R}^{N} \backslash B_{m}}|D g|^{p} d x \leq \varepsilon \int_{\mathbb{R}^{N}}|D g|^{p} d x .
$$

For $k \in \mathbb{N}$, define

$$
\mathbf{J}_{k}=\left\{Q^{\prime} \in \Omega_{k} ; Q^{\prime} \cap B_{m} \neq \varnothing\right\}
$$

Take $Q^{\prime} \in \mathbf{J}_{k}$ and $x \in Q^{\prime} \cap B_{m}$. From (4.2),

$$
\frac{1}{\left|Q^{\prime}\right|^{1 / N}} f_{Q^{\prime}}|g(y)-g(x)-\langle D g(x), y-x\rangle| d y \leq \rho_{k}(x),
$$

and from (4.3),

$$
f_{Q^{\prime}}|D g(y)-D g(x)|^{p} d y \leq \tau_{k}(x)
$$


Since $\rho_{n}$ and $\tau_{n}$ go to zero uniformly on $B_{m}$, it follows from (4.6) and (4.7) that there exists $k$ such that if $Q^{\prime} \in \mathbf{J}_{k}$ and $x \in Q^{\prime} \cap B_{m}$, then

$$
\left|\left\{y \in Q^{\prime} ;|g(y)-g(x)-D g(x)(y-x)|>\frac{\delta_{1}}{2 m}\left|Q^{\prime}\right|^{1 / N}\right\}\right|<\frac{\delta_{2}}{2}\left|Q^{\prime}\right|
$$

and

$$
|D g(x)|^{p}\left|Q^{\prime}\right| \geq(1-\varepsilon) \int_{Q^{\prime}}|D g|^{p} d y .
$$

Since $g_{\delta}$ converges to $g$ in measure, we have

$$
\left|\left\{y \in Q^{\prime} ;\left|g_{\delta}(y)-g(y)\right|>\frac{\delta_{1}}{2 m}\left|Q^{\prime}\right|^{1 / N}\right\}\right|<\frac{\delta_{2}}{2}\left|Q^{\prime}\right|,
$$

as $\delta$ is small. We deduce from (4.8) and (4.10) that

$$
\left|\left\{y \in Q^{\prime} ;\left|g_{\delta}(y)-g(x)-D g(x)(y-x)\right|>\frac{\delta_{1}}{m}\left|Q^{\prime}\right|^{1 / N}\right\}\right|<\delta_{2}\left|Q^{\prime}\right|,
$$

as $\delta$ is small. Applying Lemma 8, we obtain

$$
\varliminf_{\delta \rightarrow 0} \iint_{\left|g_{\delta}(x)-g_{\delta}(y)\right|>\delta} \frac{\delta^{p}}{|x-y|^{N+p}} d x d y \geq\left(C_{N, p}-\varepsilon\right)|D g(x)|^{p}\left|Q^{\prime}\right|,
$$

which implies, by (4.9),

$$
\varliminf_{\delta \rightarrow 0} \iint_{g_{\delta \delta}(x)-g_{\delta}(y) \mid>\delta} \frac{\delta^{p}}{|x-y|^{N+p}} d x d y \geq\left(C_{N, p}-\varepsilon\right)(1-\varepsilon) \int_{Q^{\prime}}|D g|^{p} d y .
$$

Since

$$
\frac{\lim _{\delta \rightarrow 0}}{\int_{\substack{\mathbb{R}^{N} \\\left|g_{\delta}(x)-g_{\delta}(y)\right|>\delta}}} \frac{\delta^{p}}{|x-y|^{N+p}} d x d y \geq \sum_{Q^{\prime} \in \mathbf{J}_{k}} \frac{\lim _{\delta \rightarrow 0}}{\delta \rightarrow \int_{\left|g_{\delta}(x)-g_{\delta}(y)\right|>\delta}} \frac{\delta^{p}}{|x-y|^{N+p}} d x d y,
$$

it follows from (4.5) and (4.11) that

$$
\varliminf_{\delta \rightarrow 0} \int_{\substack{\mathbb{R}^{N} \times \mathbb{R}^{N} \\\left|g_{\delta}(x)-g_{\delta}(y)\right|>\delta}} \frac{\delta^{p}}{|x-y|^{N+p}} d x d y \geq\left(C_{N, p}-\varepsilon\right)(1-\varepsilon)^{2} \int_{\mathbb{R}^{N}}|D g|^{p} d x .
$$


Since $\varepsilon>0$ is arbitrary,

$$
\varliminf_{\delta \rightarrow 0} \iint_{\substack{\mathbb{R}^{N} \times \mathbb{R}^{N} \\\left|g_{\delta}(x)-g_{\delta}(y)\right|>\delta}} \frac{\delta^{p}}{|x-y|^{N+p}} d x d y \geq C_{N, p} \int_{\mathbb{R}^{N}}|D g|^{p} d x .
$$

\section{A characterization of $B V$ functions}

In this section, we introduce a characterization of $\mathrm{BV}$ functions which is useful in the proof of Proposition 2 in the case $p=1$. As mentioned in the introduction, this characterization is motivated from the one based on the notion of essential variation on almost every line. We first present the following notion, which is motivated by the concept of Lebesgue points.

\section{Definition 4}

Let $g \in L^{1}\left(\prod_{i=1}^{N}\left(a_{i}, b_{i}\right)\right)\left(a_{i}<b_{i}\right)$, and let $t \in\left(a_{1}, b_{1}\right)$. Then the surface $x_{1}=t$ is said to be a Lebesgue surface of $g$ if and only if for almost every $z^{\prime} \in \prod_{i=2}^{N}\left(a_{i}, b_{i}\right)$, $\left(t, z^{\prime}\right)$ is a Lebesgue point of $g$, the restriction of $g$ on the surface $x_{1}=t$ is integrable with respect to $(N-1)$-Hausdorff measure, and

$$
\lim _{\varepsilon \rightarrow 0_{+}} \int_{t-\varepsilon}^{t+\varepsilon} \int_{\prod_{i=2}^{N}\left(a_{i}, b_{i}\right)}\left|g\left(s, z^{\prime}\right)-g\left(t, z^{\prime}\right)\right| d z^{\prime} d s=0 .
$$

For $i=2, \ldots, N$, we also define the notion of the Lebesgue surface for surfaces $x_{i}=t$ with $t \in\left(a_{i}, b_{i}\right)$ in the similar manner.

The following lemma is a consequence of the theory of maximal functions (see, e.g., [20]) and Fubini's theorem. The details of the proof are left to the reader.

\section{LEMMA 9}

Let $g \in L^{1}\left(\prod_{i=1}^{N}\left(a_{i}, b_{i}\right)\right)$, and let $j \in\{1, \ldots, N\}$. Then for almost every $t \in\left(a_{j}, b_{j}\right)$, the surface $x_{j}=t$ is a Lebesgue surface of $g$.

The following definition is used in Proposition 3, which deals with a characterization for BV functions.

\section{Definition 5}

Let $g \in L^{1}\left(\prod_{i=1}^{N}\left(a_{i}, b_{i}\right)\right)$. The essential variation of $g$ in the first direction is defined 
as

$$
\operatorname{ess} V(g, 1)=\sup \left\{\sum_{i=1}^{m} \int_{\prod_{i=2}^{N}\left[a_{i}, b_{i}\right]}\left|g\left(t_{i+1}, x^{\prime}\right)-g\left(t_{i}, x^{\prime}\right)\right| d x^{\prime}\right\}
$$

where the supremum is taken over all finite partitions $\left\{a_{1}<t_{1}<\cdots<t_{m+1}<b_{1}\right\}$ such that the surface $x_{1}=t_{k}$ is a Lebesgue surface of $g$ for $1 \leq k \leq m+1$. For $2 \leq j \leq N$, we also define ess $V(g, j)$ the essential variation of $g$ in the $j$ th direction in the similar manner.

The following proposition, which gives a characterization for $B V$ functions, is the main result of this section.

PROPOSITION 3

Let $g \in L^{1}\left(\prod_{i=1}^{N}\left(a_{i}, b_{i}\right)\right)$. Then $g \in \mathrm{BV}\left(\prod_{i=1}^{N}\left(a_{i}, b_{i}\right)\right)$ if and only if

$$
\text { ess } V(g, j)<+\infty, \quad \forall 1 \leq j \leq N .
$$

Moreover, for $g \in \mathrm{BV}\left(\prod_{i=1}^{N}\left(a_{i}, b_{i}\right)\right)$,

$$
\text { ess } V(g, j)=\left\|D g \cdot e_{j}\right\|\left(\prod_{i=1}^{N}\left(a_{i}, b_{i}\right)\right), \quad \forall 1 \leq j \leq N .
$$

Here $D g \cdot e_{j}$ denotes the derivative of $g$ with respect to the variable $x_{j}$, and $\| D g$. $e_{j} \|\left(\prod_{i=1}^{N}\left(a_{i}, b_{i}\right)\right)$ denotes its total mass.

\section{Remark 2}

This characterization is well known in the case $N=1,2$.

\section{Proof}

The proof we present below is quite standard. Suppose that ess $V(g, j)<+\infty$ for all $1 \leq j \leq N$. We claim that $g \in \mathrm{BV}\left(\prod_{i=1}^{N}\left(a_{i}, b_{i}\right)\right)$ and

$$
\left\|D g \cdot e_{j}\right\|\left(\prod_{i=1}^{N}\left(a_{i}, b_{i}\right)\right) \leq \operatorname{ess} V(g, j), \quad \forall 1 \leq j \leq N .
$$

Let $\left(\rho_{\varepsilon}\right)$ be a standard sequence of smooth mollifiers on $\mathbb{R}$ such that supp $\rho_{\varepsilon} \subset(-\varepsilon, \varepsilon)$. Fix $\varepsilon>0$, and set $g_{\varepsilon}(x)=\int_{\mathbb{R}} g\left(x_{1}-s, x^{\prime}\right) \rho_{\varepsilon}(s) d s$. Choose arbitrarily $a_{1}+\varepsilon<t_{1}<$ 
$\cdots<t_{m+1}<b_{1}-\varepsilon$. We have

$$
\begin{aligned}
& \sum_{k=1}^{m} \int_{\prod_{i=2}^{N}\left[a_{i}, b_{i}\right]}\left|g_{\varepsilon}\left(t_{k+1}, x^{\prime}\right)-g_{\varepsilon}\left(t_{k}, x^{\prime}\right)\right| d x^{\prime} \\
& \quad=\sum_{k=1}^{m} \int_{\prod_{i=2}^{N}\left[a_{i}, b_{i}\right]}\left|\int_{-\varepsilon}^{\varepsilon} \rho_{\varepsilon}(s)\left[g\left(t_{k+1}-s, x^{\prime}\right)-g\left(t_{k}-s, x^{\prime}\right)\right] d s\right| d x^{\prime} \\
& \quad \leq \sum_{k=1}^{m} \int_{\prod_{i=2}^{N}\left[a_{i}, b_{i}\right]} \int_{-\varepsilon}^{\varepsilon} \rho_{\varepsilon}(s)\left|g\left(t_{k+1}-s, x^{\prime}\right)-g\left(t_{k}-s, x^{\prime}\right)\right| d s d x^{\prime} \\
& \quad \leq \int_{-\varepsilon}^{\varepsilon} \rho_{\varepsilon}(s) \sum_{k=1}^{m} \int_{\prod_{i=2}^{N}\left[a_{i}, b_{i}\right]}\left|g\left(t_{k+1}-s, x^{\prime}\right)-g\left(t_{k}-s, x^{\prime}\right)\right| d x^{\prime} d s .
\end{aligned}
$$

On the other hand, for almost every $t \in\left(a_{1}, b_{1}\right)$, the surface $x_{1}=t$ is a Lebesgue surface of $g$ (see Lemma 9). Hence

$$
\begin{gathered}
\int_{-\varepsilon}^{\varepsilon} \rho_{\varepsilon}(s) \sum_{k=1}^{m} \int_{\prod_{i=2}^{N}\left[a_{i}, b_{i}\right]}\left|g\left(t_{k+1}-s, x^{\prime}\right)-g\left(t_{k}-s, x^{\prime}\right)\right| d x^{\prime} d s \\
\leq \int_{-\varepsilon}^{\varepsilon} \rho_{\varepsilon}(s) \operatorname{ess} V(g, 1) d s \leq \operatorname{ess} V(g, 1) .
\end{gathered}
$$

Combining (5.3) and (5.4) yields

$$
\int_{a_{1}+\varepsilon}^{b_{1}-\varepsilon} \int_{\prod_{i=2}^{N}\left[a_{i}, b_{i}\right]}\left|D g_{\varepsilon} \cdot e_{1}\right| d x^{\prime} d s \leq \operatorname{ess} V(g, 1) .
$$

This implies

$$
\left\|D g \cdot e_{1}\right\|\left(\prod_{i=1}^{N}\left(a_{i}, b_{i}\right)\right) \leq \operatorname{ess} V(g, 1)
$$

Similarly,

$$
\left\|D g \cdot e_{j}\right\|\left(\prod_{i=1}^{N}\left(a_{i}, b_{i}\right)\right) \leq \operatorname{ess} V(g, j), \quad \forall 2 \leq j \leq N
$$

Therefore, $g \in \mathrm{BV}\left(\prod_{i=1}^{N}\left(a_{i}, b_{i}\right)\right)$ and (5.2). 
We now suppose that $g \in \mathrm{BV}\left(\prod_{i=1}^{N}\left(a_{i}, b_{i}\right)\right)$. We claim that

$$
\text { ess } V(g, j) \leq\left\|D g \cdot e_{j}\right\|\left(\prod_{i=1}^{N}\left(a_{i}, b_{i}\right)\right), \quad \forall 1 \leq j \leq N
$$

In fact, consider $\left\{a_{1}<t_{1}<\cdots<t_{m+1}<b_{1}\right\}$ such that the surface $x_{1}=t_{k}$ is a Lebesgue surface of $g$ for $k=1, \ldots, m+1$. Then, by (5.1),

$$
\begin{aligned}
& \sum_{k=1}^{m} \int_{\prod_{i=2}^{N}\left[a_{i}, b_{i}\right]}\left|g\left(t_{k+1}, x^{\prime}\right)-g\left(t_{k}, x^{\prime}\right)\right| d x^{\prime} \\
& \quad=\lim _{\varepsilon \rightarrow 0} \sum_{k=1}^{m} \int_{\prod_{i=2}^{N}\left[a_{i}, b_{i}\right]}\left|g_{\varepsilon}\left(t_{k+1}, x^{\prime}\right)-g_{\varepsilon}\left(t_{k}, x^{\prime}\right)\right| d x^{\prime} .
\end{aligned}
$$

However,

$$
\sum_{k=1}^{m} \int_{\prod_{i=2}^{N}\left[a_{i}, b_{i}\right]}\left|g_{\varepsilon}\left(t_{k+1}, x^{\prime}\right)-g_{\varepsilon}\left(t_{k}, x^{\prime}\right)\right| d x^{\prime} \leq \int_{t_{1}}^{t_{m+1}} \int_{\prod_{i=2}^{N}\left[a_{i}, b_{i}\right]}\left|D g_{\varepsilon} \cdot e_{1}\right| d x
$$

and

$$
\int_{t_{1}}^{t_{m+1}} \int_{\prod_{i=2}^{N}\left[a_{i}, b_{i}\right]}\left|D g_{\varepsilon} \cdot e_{1}\right| d x \leq\left\|D g \cdot e_{1}\right\|\left(\prod_{i=1}^{N}\left(a_{i}, b_{i}\right)\right)
$$

when $\varepsilon$ is small. It follows that

$$
\sum_{k=1}^{m} \int_{\prod_{i=2}^{N}\left[a_{i}, b_{i}\right]}\left|g\left(t_{k+1}, x^{\prime}\right)-g\left(t_{k}, x^{\prime}\right)\right| d x^{\prime} \leq\left\|D g \cdot e_{1}\right\|\left(\prod_{i=1}^{N}\left(a_{i}, b_{i}\right)\right),
$$

which implies

$$
\operatorname{ess} V(g, 1) \leq\left\|D g \cdot e_{1}\right\|\left(\prod_{i=1}^{N}\left(a_{i}, b_{i}\right)\right)
$$

Similarly,

$$
\operatorname{ess} V(g, j) \leq\left\|D g \cdot e_{j}\right\|\left(\prod_{i=1}^{N}\left(a_{i}, b_{i}\right)\right), \quad \forall 2 \leq j \leq N .
$$

Thus (5.5) is proved. 
The conclusion of Proposition 3 now follows from (5.2) and (5.5).

\section{Remark 3}

We do not use any property of Lebesgue points in the definition of Lebesgue surfaces in the proof, but it will be useful in the proof of Proposition 2 in the case $p=1$ (see the proof of Lemma 14).

\section{Proof of Proposition 2 in the case $p=1$}

\subsection{Another definition of $C_{N, 1}$}

Define

$$
\mathbf{b}_{N, 1}:=\inf \varliminf_{\delta \rightarrow 0} \iint_{\left|g_{\delta}(x)-g_{\delta}(y)\right|>\delta} \frac{\delta}{|x-y|^{N+1}} d x d y,
$$

where the infimum is taken over all family of measurable functions $\left(g_{\delta}\right)_{\delta \in(0,1)}$ such that $g_{\delta}$ converges to $H_{1 / 2}$ in measure as $\delta$ goes to zero. Here and afterward $H_{c}(x):=$ $H\left(x_{1}-c, x^{\prime}\right)$ for any $c \in \mathbb{R}$, where $H$ is the function defined on $\mathbb{R}^{N}$ by

$$
H(x)= \begin{cases}0 & \text { if } x_{1}<0 \\ 1 & \text { otherwise }\end{cases}
$$

This section is devoted to proving the following proposition.

PROPOSITION 4

We have

$$
\mathbf{b}_{N, 1}=C_{N, 1} \text {. }
$$

The proof of Proposition 4 is based on two lemmas. The first one is used to prove that $\mathbf{b}_{N, 1} \geq C_{N, 1}$.

\section{LEMMA 10}

There exist a sequence of measurable functions $\left(\psi_{k}\right)$ and a sequence of positive numbers $\left(\tau_{k}\right)$ converging to zero such that $\psi_{k}$ converges to $g \equiv x_{1}$ in measure on $Q$, and

$$
\varliminf_{k \rightarrow \infty} \iint_{\substack{Q^{2} \\\left|\psi_{k}(x)-\psi_{k}(y)\right|>\tau_{k}}} \frac{\tau_{k}}{|x-y|^{N+1}} d x d y=\mathbf{b}_{N, 1} .
$$


Proof

From the definition of $\mathbf{b}_{N, 1}$, there exist a sequence $\left(\delta_{k}\right)$ converging to zero and a sequence of measurable functions $\left(g_{k}\right)$ converging in measure to $H_{1 / 2}$ as $k$ goes to infinity such that

$$
\lim _{k \rightarrow \infty} \iint_{\substack{Q^{2} \\\left|g_{k}(x)-g_{k}(y)\right|>\delta_{k}}} \frac{\delta_{k}}{|x-y|^{N+1}} d x d y=\mathbf{b}_{N, 1} .
$$

Since $g_{k}$ converges to $H_{1 / 2}$ in measure on $Q$, there exists a sequence of positive numbers $\left(c_{k}\right)_{k \in \mathbb{N}}$ converging to zero such that

$$
\lim _{k \rightarrow \infty} \frac{\left|\left\{x \in Q ;\left|g_{k}(x)-H_{1 / 2}(x)\right| \geq c_{k}\right\}\right|}{c_{k}}=0 .
$$

Define $h_{1, k}, h_{2, k}: Q \rightarrow \mathbb{R}$ as follows:

$$
h_{1, k}(x)= \begin{cases}c_{k} & \text { if } x_{1}<\frac{1}{2}-c_{k}, \\ 1+c_{k} & \text { if } x_{1}>\frac{1}{2}, \\ \frac{1}{c_{k}}\left(x_{1}-\frac{1}{2}+c_{k}\right)+c_{k} & \text { otherwise }\end{cases}
$$

and

$$
h_{2, k}(x)= \begin{cases}-c_{k} & \text { if } x_{1}<\frac{1}{2}, \\ 1-c_{k} & \text { if } x_{1}>\frac{1}{2}+c_{k}, \\ \frac{1}{c_{k}}\left(x_{1}-\frac{1}{2}\right)-c_{k} & \text { otherwise }\end{cases}
$$

Set

$$
g_{1, k}=\min \left(\max \left(g_{k}, h_{2, k}\right), h_{1, k}\right)
$$

and

$$
g_{2, k}=\min \left(\max \left(g_{1, k}, c_{k}\right), 1-c_{k}\right) .
$$

We claim that

$$
\begin{aligned}
g_{2, k}(x) & =c_{k}, \quad \forall x \in Q, x_{1}<\frac{1}{2}-c_{k}, \quad g_{2, k}(x)=1-c_{k}, \quad \forall x \in Q, \\
x_{1} & >\frac{1}{2}+c_{k},
\end{aligned}
$$


and

$$
\lim _{k \rightarrow \infty} \iint_{\substack{Q^{2} \\\left|g_{2, k}(x)-g_{2, k}(y)\right|>\delta_{k}}} \frac{\delta_{k}}{|x-y|^{N+1}} d x d y=\mathbf{b}_{N, 1} .
$$

In fact, it suffices to prove (6.5). By Corollary 1, we have

$$
\iint_{Q^{2}} \frac{\delta_{k}}{|x-y|^{N+1}} d x d y \leq \iint_{\left|g_{2, k}(x)-g_{2, k}(y)\right|>\delta_{k}} \frac{\delta_{k}}{|x-y|^{N+1}} d x d y .
$$

Since $\left|D h_{1, k}(x)\right| \leq 1 / c_{k}$ and $\left|D h_{2, k}(x)\right| \leq 1 / c_{k}$ for all $x \in Q$, it then follows from (6.3) and Lemma 1 that

$$
\varlimsup_{k \rightarrow \infty} \iint_{\left|g_{1, k}(x)-g_{1, k}(y)\right|>\delta_{k}} \frac{\delta_{k}}{|x-y|^{N+1}} d x d y \leq \varlimsup_{k \rightarrow \infty} \iint_{\left|g_{k}(x)-g_{k}(y)\right|>\delta_{k}} \frac{\delta_{k}}{|x-y|^{N+1}} d x d y .
$$

Combining (6.2), (6.6), and (6.7) yields

$$
\varlimsup_{k \rightarrow \infty} \iint_{\substack{Q^{2} \\\left|g_{2, k}(x)-g_{2, k}(y)\right|>\delta_{k}}} \frac{\delta_{k}}{|x-y|^{N+1}} d x d y \leq \mathbf{b}_{N, 1} .
$$

From (6.8) and (6.1) we obtain (6.5).

Let $h_{k}: Q \mapsto \mathbb{R}$ be defined by

$$
h_{k}(x)=\frac{g_{2, k}(x)-c_{k}}{1-2 c_{k}},
$$

and set $\varepsilon_{k}=\delta_{k} /\left(1-2 c_{k}\right)$. It is clear that $\varepsilon_{k}$ converges to zero as $k$ goes to infinity, and

$$
\varlimsup_{k \rightarrow \infty} \iint_{\left|h_{k}(x)-h_{k}(y)\right|>\varepsilon_{k}} \frac{\varepsilon_{k}}{|x-y|^{N+1}} d x d y=\varlimsup_{k \rightarrow \infty} \iint_{\left|g_{k}(x)-g_{k}(y)\right|>\delta_{k}} \frac{\delta_{k}}{|x-y|^{N+1}} d x d y .
$$

We deduce from (6.5) and (6.10) that

$$
\varlimsup_{k \rightarrow \infty} \iint_{\left|h_{k}(x)-h_{k}(y)\right|>\varepsilon_{k}} \frac{\varepsilon_{k}}{|x-y|^{N+1}} d x d y=\mathbf{b}_{N, 1} .
$$

For each $n \in \mathbb{N}$ (arbitrary), consider the sequence $\left(f_{k}\right): Q \mapsto \mathbb{R}$ which is defined as follows:

$$
f_{k}(x)=\frac{1}{n} h_{k}\left(x_{1}-\frac{i}{n}+\frac{1}{2}-\frac{1}{2 n}, x^{\prime}\right)+\frac{i}{n} \quad \text { if } x_{1} \in\left[\frac{i}{n}, \frac{i+1}{n}\right], 0 \leq i \leq n-1 .
$$


We claim that

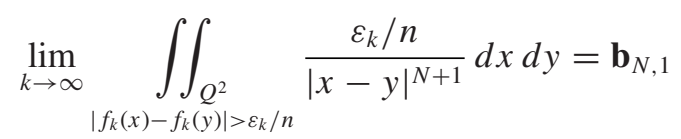

and

$$
\int_{Q}\left|f_{k}(x)-x_{1}\right| d x \leq \frac{1}{n}
$$

Indeed, (6.13) is clear from the definition of $f_{k}$ and the fact that $0 \leq h_{k}(x) \leq 1$ for all $x \in Q$. It suffices to prove (6.12). We have

$$
\begin{aligned}
& \iint_{Q^{2}} \frac{\varepsilon_{k} / n}{|x-y|^{N+1}} d x d y=\sum_{i=0}^{n-1} \int_{\substack{x_{1} \in[i / n,(i+1) / n] \\
y_{1} \in[i / n,(i+1) / n] \\
\left|f_{k}(x)-f_{k}(y)\right|>\varepsilon_{k} / n}} \frac{\varepsilon_{k} / n}{|x-y|^{N+1}} d x d y \\
& +\sum_{i=0}^{n-1} \int_{\substack{\left.x_{1} \in[i / n,(i+1) / n] \\
y_{1} \notin i / n,(i+1) / n\right] \\
\left|f_{k}(x)-f_{k}(y)\right|>\varepsilon_{k} / n}} \frac{\varepsilon_{k} / n}{|x-y|^{N+1}} d x d y .
\end{aligned}
$$

On the other hand, since $h_{k}(x)=0$ if $x_{1}<1 / 2-c_{k}, h_{k}(x)=1$ if $x_{1}>1 / 2+c_{k}$, and $c_{k}$ converges to zero as $k$ goes to infinity (by (6.4) and (6.9)), it follows from (6.11) that

$$
\begin{gathered}
\iint_{\substack{Q^{2} \\
x_{1} \in[i / n,(i+1) / n] \\
y_{1} \in[i / n,(i+1) / n] \\
f_{k}(x)-f_{k}(y) \mid>\varepsilon_{k} / n}} \frac{\varepsilon_{k} / n}{|x-y|^{N+1}} d x d y=\frac{1}{n} \int_{\substack{x_{1} \in[1 / 2-1 / 2 n, 1 / 2+1 / 2 n] \\
y_{1} \in[1 / 2-1 / 2 n, 1 / 2+1 / 2 n] \\
\left|h_{k}(x)-h_{k}(y)\right|>\varepsilon_{k}}} \frac{\varepsilon_{k}}{|x-y|^{N+1}} d x d y \\
\lim _{k \rightarrow \infty} \iint_{\substack{Q_{1}^{2} \in[1 / 2-1 / 2 n, 1 / 2+1 / 2 n] \\
y_{1} \in[1 / 2-1 / 2 n, 1 / 2+1 / 2 n] \\
\left|h_{k}(x)-h_{k}(y)\right|>\varepsilon_{k}}} \frac{\varepsilon_{k}}{|x-y|^{N+1}} d x d y=\mathbf{b}_{N, 1}
\end{gathered}
$$

and

$$
\lim _{k \rightarrow \infty} \int_{\substack{x_{1} \in[i / n,(i+1) / n] \\ y_{1} \notin[i / n,(i+1) / n] \\\left|f_{k}(x)-f_{k}(y)\right|>\varepsilon_{k} / n}} \frac{\varepsilon_{k} / n}{|x-y|^{N+1}} d x d y=0 .
$$


Combining (6.14) - (6.17) yields (6.12)

The conclusion now follows from (6.12) and (6.13).

We also have the following result, which implies $C_{N, 1} \geq \mathbf{b}_{N, 1}$.

\section{LEMMA 11}

There exist a sequence of measurable functions $\left(\psi_{k}\right)$ and a sequence of positive numbers $\left(\tau_{k}\right)$ converging to zero such that $\psi_{k}$ converges to $H_{1 / 2}$ in measure on $Q$ and

$$
\varliminf_{k \rightarrow \infty} \iint_{\left|\psi_{k}(x)-\psi_{k}(y)\right|>\tau_{k}} \frac{\tau_{k}}{|x-y|^{N+1}} d x d y=C_{N, 1} .
$$

Proof

Let $\left(g_{n}\right)$ be a sequence of functions defined on $Q$ as follows:

$$
g_{n}(x)= \begin{cases}0 & \text { if } x_{1} \leq \frac{1}{2}-\frac{1}{n}, \\ n\left[x_{1}-\frac{1}{2}+\frac{1}{n}\right] & \text { if } \frac{1}{2}-\frac{1}{n}<x_{1} \leq \frac{1}{2}, \\ 1 & \text { otherwise, } \quad \forall n \in \mathbb{N} .\end{cases}
$$

For each $n$, by Lemma 4 , there exists a family of measurable functions $\left(g_{n, \delta}\right)_{\delta \in(0,1)}$ defined on $Q$ such that $g_{n, \delta}$ converges to $g_{n}$ in measure and

$$
\lim _{\delta \rightarrow 0} \iint_{\substack{Q^{2} \\\left|g_{n, \delta}(x)-g_{n, \delta}(y)\right|>\delta}} \frac{\delta}{|x-y|^{N+1}} d x d y=C_{N, 1} .
$$

Therefore, the conclusion follows.

We are ready to give the following.

Proof of Proposition 4

We have $C_{N, 1} \leq \mathbf{b}_{N, 1}$ by Lemma 10 and the definition of $C_{N, 1}$, and we have $C_{N, 1} \geq \mathbf{b}_{N, 1}$ by Lemma 11 and the definition of $\mathbf{b}_{N, 1}$. This implies $\mathbf{b}_{N, 1}=$ $C_{N, 1}$.

\subsection{Some useful lemmas}

In this section, we prove some useful lemmas which we use in the proof of Proposition 2 in the case $p=1$. Our main goal is to prove Lemma 14. From the definition of $\mathbf{b}_{N, 1}$, we have the following.

LEMMA 12

For any $\varepsilon>0$, there exist three positive numbers $\delta_{1}, \delta_{2}$, and $\delta_{3}$ such that if $g \in$ 


$$
\begin{aligned}
& L^{1}\left(\prod_{i=1}^{N}\left[a_{i}, b_{i}\right]\right)\left(a_{i}<b_{i}\right), \\
& \quad\left|\left\{x \in \prod_{i=1}^{N}\left[a_{i}, b_{i}\right] ;\left|g(x)-\left(c H_{a_{1}+\left(b_{1}-a_{1}\right) / 2}(x)+d\right)\right|>|c| \delta_{1}\right\}\right|<\delta_{2} \prod_{i=1}^{N}\left(b_{i}-a_{i}\right),
\end{aligned}
$$

and $\delta<|c| \delta_{3}$, for some $c$ and $d$ in $\mathbb{R}$, then

$$
\iint_{\substack{\prod_{i=1}^{N}\left[a_{i}, b_{i}\right] \times \prod_{i=1}^{N}\left[g(x)-g(y)|>\delta\\| a_{i}, b_{i}\right]}} \frac{\delta}{|x-y|^{N+1}} d x d y \geq|c|\left(\mathbf{b}_{N, 1}-\varepsilon\right) .
$$

\section{Proof}

The proof is similar to that of Lemma 8. The details are left to the reader.

The following lemma, in which we do not require any condition on the convergence of $\left(g_{\delta}\right)$, plays an important role in the proof of Lemma 14.

\section{LEMMA 13}

Let $\left(g_{\delta}\right)_{\delta \in(0,1)} \subset L^{1}\left(\prod_{i=1}^{N}\left[a_{i}, b_{i}\right]\right)$. Assume that $g_{\delta}(x) \leq 0$ for $x$ with $x_{1} \leq a_{1}+\delta$ and $g_{\delta}(x) \geq c(c>0)$ for $x$ such that $x_{1} \geq b_{1}-\delta$. Then

$$
\frac{\lim _{\delta \rightarrow 0}}{\substack{\prod_{i=1}^{N}\left[a_{i}, b_{i}\right] \times \prod_{i=1}^{N}\left[a_{i}, b_{i}\right] \\|g(x)-g(y)|>\delta}} \frac{\delta}{|x-y|^{N+1}} d x d y \geq c \mathbf{b}_{N, 1} .
$$

\section{Proof}

Without loss of generality, by Corollary 1 , one may assume that $g_{\delta}(x)=0$ for all $x$ such that $x_{1}<a_{1}+\delta$ and $g(x)=c$ for all $x$ such that $x_{1} \geq b_{1}-\delta$. For $\varepsilon>0$, let $\delta_{2}$ be a positive constant corresponding to $\varepsilon$ in Lemma 12. Set $Q_{\delta}:=$ $\left[a_{1}-\left(b_{1}-a_{1}\right) / 2 \delta_{2}, b_{1}+\left(b_{1}-a_{1}\right) / 2 \delta_{2}\right] \times \prod_{i=2}^{N}\left[a_{i}, b_{i}\right]$. Define $h_{\delta}: Q_{\delta} \mapsto \mathbb{R}$ by

$$
h_{\delta}(x)= \begin{cases}0 & \text { if } x_{1} \in\left(a_{1}-\frac{b_{1}-a_{1}}{2 \delta_{2}}, a_{1}\right), \\ g_{\delta}(x) & \text { if } x_{1} \in\left(a_{1}, b_{1}\right), \\ c & \text { if } x_{1} \in\left(b_{1}, b_{1}+\frac{b_{1}-a_{1}}{2 \delta_{2}}\right) .\end{cases}
$$

Applying Lemma 12 for the function $h_{\delta}$, we have

$$
\varliminf_{\delta \rightarrow 0} \iint_{\substack{Q_{\delta}^{2} \\\left|h_{\delta}(x)-h_{\delta}(y)\right|>\delta}} \frac{\delta}{|x-y|^{N+1}} d x d y \geq c\left(\mathbf{b}_{N, 1}-\varepsilon\right) .
$$


Since $g_{\delta}(x)=0$ if $x_{1}<a_{1}+\delta$ and $g_{\delta}(x)=c$ if $x_{1}>b_{1}-\delta$, it follows that

$$
\varliminf_{\delta \rightarrow 0} \iint_{\substack{\prod_{i=1}^{N}\left[a_{i}, b_{i}\right] \times \prod_{i=1}^{N}\left[a_{i}, b_{i}\right] \\\left|g_{\delta}(x)-g_{\delta}(y)\right|>\delta}} \frac{\delta}{|x-y|^{N+1}} d x d y \geq c\left(\mathbf{b}_{N, 1}-\varepsilon\right) .
$$

Since $\varepsilon>0$ is arbitrary, the conclusion follows.

The following lemma plays a crucial role in the proof of Proposition 2 in the case $p=1$.

\section{LEMMA 14}

Let $g \in L^{1}\left(\prod_{i=1}^{N}\left(a_{i}, b_{i}\right)\right)$, and let $\left(g_{\delta}\right)_{\delta \in(0,1)} \subset L^{1}\left(\prod_{i=1}^{N}\left[a_{i}, b_{i}\right]\right)\left(a_{i}<b_{i}\right)$ be such that $g_{\delta}$ converges to $g$ in measure on $\prod_{i=1}^{N}\left[a_{i}, b_{i}\right]$. Then for any $t_{1}$ and $t_{2}$ in $\left(a_{1}, b_{1}\right)$ $\left(t_{1}<t_{2}\right)$ such that the surface $x_{1}=t_{j}(j=1,2)$ is a Lebesgue surface of $g$, we have

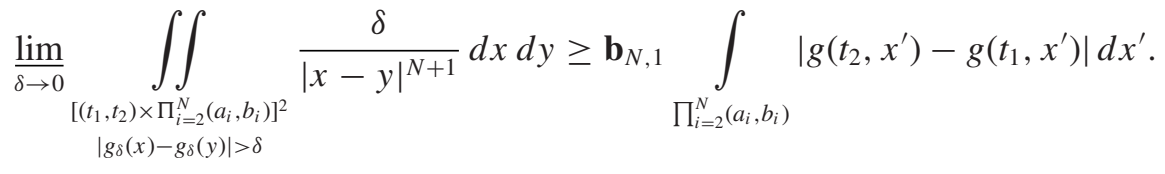

Proof

Fix $\tau>0$ (arbitrary). Let $A$ be the set of all elements $z^{\prime} \in \prod_{i=2}^{N}\left[a_{i}, b_{i}\right]$ such that $\left(t_{1}, z^{\prime}\right)$ is a Lebesgue point of $g_{\mid x_{1}=t_{1}},\left(t_{2}, z^{\prime}\right)$ is a Lebesgue point of $g_{\mid x_{1}=t_{2}} ;\left(t_{1}, z^{\prime}\right)$, and $\left(t_{2}, z^{\prime}\right)$ are Lebesgue points of $g$. For each $z^{\prime} \in A$, let $Q^{\prime}\left(z^{\prime}\right) \subset \mathbb{R}^{N-1}$ be a closed cube center at $z^{\prime}$ such that

$$
\left\{\begin{array}{l}
\text { (i) }\left|\left\{y^{\prime} \in Q^{\prime}\left(z^{\prime}\right) ;\left|g\left(t_{j}, y^{\prime}\right)-g\left(t_{j}, z^{\prime}\right)\right| \geq \tau / 2\right\}\right| \leq \tau\left|Q^{\prime}\left(z^{\prime}\right)\right|, \\
\text { (ii) } f_{Q^{\prime}\left(z^{\prime}\right)}\left|g\left(t_{j}, y^{\prime}\right)-g\left(t_{j}, z^{\prime}\right)\right| d y^{\prime} \leq \tau, \quad \text { for } j=1,2,
\end{array}\right.
$$

and

$$
\left\{\begin{array}{l}
\left|\left\{\left(x_{1}, y^{\prime}\right) \in\left(t_{1}, t_{1}+2 l\right) \times Q^{\prime}\left(z^{\prime}\right) ;\left|g\left(x_{1}, y^{\prime}\right)-g\left(t_{1}, z^{\prime}\right)\right| \geq \tau / 2\right\}\right| \leq \tau l\left|Q^{\prime}\left(z^{\prime}\right)\right|, \\
\left|\left\{\left(x_{1}, y^{\prime}\right) \in\left(t_{2}-2 l, t_{2}\right) \times Q^{\prime}\left(z^{\prime}\right) ;\left|g\left(x_{1}, y^{\prime}\right)-g\left(t_{2}, z^{\prime}\right)\right| \geq \tau / 2\right\}\right| \leq \tau l\left|Q^{\prime}\left(z^{\prime}\right)\right|,
\end{array}\right.
$$

where $l=1 / 2\left|Q^{\prime}\left(z^{\prime}\right)\right|^{1 /(N-1)}$. Hereafter in the proof, $\left|Q^{\prime}\left(z^{\prime}\right)\right|$ denotes the $(N-1)$ dimensional Hausdorff measure of $Q^{\prime}\left(z^{\prime}\right)$. 
Since $g_{\delta}$ converges to $g$ in measure, it follows from (6.19) that, when $\delta$ is small,

(i) $\left|\left\{\left(x_{1}, y^{\prime}\right) \in\left(t_{1}, t_{1}+2 l\right) \times Q^{\prime}\left(z^{\prime}\right) ;\left|g_{\delta}\left(x_{1}, y^{\prime}\right)-g\left(t_{1}, z^{\prime}\right)\right| \geq \tau\right\}\right| \leq 2 \tau l\left|Q^{\prime}\left(z^{\prime}\right)\right|$,

(ii) $\left|\left\{\left(x_{1}, y^{\prime}\right) \in\left(t_{2}-2 l, t_{2}\right) \times Q^{\prime}\left(z^{\prime}\right) ;\left|g_{\delta}\left(x_{1}, y^{\prime}\right)-g\left(t_{2}, z^{\prime}\right)\right| \geq \tau\right\}\right| \leq 2 \tau l\left|Q^{\prime}\left(z^{\prime}\right)\right|$.

We claim that

$$
\varliminf_{\delta \rightarrow 0} \iint_{\substack{\left[\left(t_{1}, t_{2}\right) \times Q^{\prime}\left(z^{\prime}\right)\right]^{2} \\\left|g_{\delta}(x)-g_{\delta}(y)\right|>\delta}} \frac{\delta}{|x-y|^{N+1}} d x d y \geq \mathbf{b}_{N, 1}\left|g\left(t_{2}, z^{\prime}\right)-g\left(t_{1}, z^{\prime}\right)\right|\left|Q^{\prime}\left(z^{\prime}\right)\right|-C \tau\left|Q^{\prime}\left(z^{\prime}\right)\right|
$$

for some constant $C$ depending only on $N$.

Without loss of generality, one may assume that $g\left(t_{1}, z^{\prime}\right)<g\left(t_{2}, z^{\prime}\right)$. Define $f_{1}:\left(t_{1}, t_{2}\right) \times Q^{\prime}\left(z^{\prime}\right) \rightarrow \mathbb{R}$ by

$$
f_{1}(y)= \begin{cases}g\left(t_{1}, z^{\prime}\right) & \text { if } y_{1} \leq t_{1}+l, \\ g\left(t_{2}, z^{\prime}\right) & \text { if } y_{1} \geq t_{1}+2 l, \\ \frac{1}{l}\left[g\left(t_{2}, z^{\prime}\right)-g\left(t_{1}, z^{\prime}\right)\right] y_{1}+2 g\left(t_{1}, z^{\prime}\right)-g\left(t_{2}, z^{\prime}\right), & \text { otherwise, }\end{cases}
$$

and set

$$
h_{1, \delta}=\min \left\{\max \left(\min \left(g_{\delta}, g\left(t_{2}, z^{\prime}\right)\right), g\left(t_{1}, z\right)\right), f_{1}\right\} .
$$

Since $f_{1}$ is a Lipschitz function with a Lipschitz constant $1 / l\left[g\left(t_{2}, z^{\prime}\right)-g\left(t_{1}, z^{\prime}\right)\right]$, it follows from (6.20(i)) and Lemma 1 that

$$
\begin{aligned}
\iint_{\substack{\left[\left(t_{1}, t_{2}\right) \times Q^{\prime}\left(z^{\prime}\right)\right]^{2} \\
\left|h_{1, \delta}(x)-h_{1, \delta}(y)\right|>\delta}} \frac{\delta}{|x-y|^{N+1}} d x d y \leq & \iint_{\substack{\left[\left(t_{1}, t_{2}\right) \times Q^{\prime}\left(z^{\prime}\right)\right]^{2} \\
\left|g_{\delta}(x)-g_{\delta}(y)\right|>\delta}} \frac{\delta}{|x-y|^{N+1}} d x d y \\
& +C \tau\left[g\left(t_{2}, z^{\prime}\right)-g\left(t_{1}, z^{\prime}\right)\right]\left|Q^{\prime}\left(z^{\prime}\right)\right| .
\end{aligned}
$$

Similarly, define $f_{2}:\left(t_{1}, t_{2}\right) \times Q^{\prime}\left(z^{\prime}\right) \rightarrow \mathbb{R}$ by

$$
f_{2}(y)= \begin{cases}g\left(t_{1}, z^{\prime}\right) & \text { if } y_{1} \leq t_{2}-2 l, \\ g\left(t_{2}, z^{\prime}\right) & \text { if } y_{1} \geq t_{2}-l \\ \frac{1}{l}\left[g\left(t_{2}, z^{\prime}\right)-g\left(t_{1}, z^{\prime}\right)\right]\left(y_{1}-t_{2}+2 l\right)+g\left(t_{1}, z^{\prime}\right) & \text { otherwise }\end{cases}
$$

and set

$$
h_{2, \delta}=\max \left(g_{1, \delta}, f_{2}\right) \text {. }
$$


We have

$$
\begin{aligned}
\iint_{\substack{\left[\left(t_{1}, t_{2}\right) \times Q^{\prime}\left(z^{\prime}\right)\right]^{2} \\
\left|g_{2, \delta}(x)-g_{2, \delta}(y)\right|>\delta}} \frac{\delta}{|x-y|^{N+1}} d x d y \leq & \iint_{\substack{\left[\left(t_{1}, t_{2}\right) \times Q^{\prime}\left(z^{\prime}\right)\right]^{2} \\
\left|g_{1, \delta}(x)-g_{1, \delta}(y)\right|>\delta}} \frac{\delta}{|x-y|^{N+1}} d x d y \\
& +C \tau\left[g\left(t_{2}, z^{\prime}\right)-g\left(t_{1}, z^{\prime}\right)\right]\left|Q^{\prime}\left(z^{\prime}\right)\right| .
\end{aligned}
$$

Combining (6.22) and (6.23) yields

$$
\begin{aligned}
\iint_{\substack{\left[\left(t_{1}, t_{2}\right) \times Q^{\prime}\left(z^{\prime}\right)\right]^{2} \\
\left|g_{2, \delta}(x)-g_{2, \delta}(y)\right|>\delta}} \frac{\delta}{|x-y|^{N+1}} d x d y \leq & \iint_{\substack{\left[\left(t_{1}, t_{2}\right) \times Q^{\prime}\left(z^{\prime}\right)\right]^{2} \\
\left|g_{\delta}(x)-g_{\delta}(y)\right|>\delta}} \frac{\delta}{|x-y|^{N+1}} d x d y \\
& +C \tau\left[g\left(t_{2}, z^{\prime}\right)-g\left(t_{1}, z^{\prime}\right)\right]\left|Q^{\prime}\left(z^{\prime}\right)\right| .
\end{aligned}
$$

On the other hand, by Lemma 13,

$$
\frac{\lim _{\delta \rightarrow 0}}{\substack{\left[\left(t_{1}, t_{2}\right) \times Q^{\prime}\left(z^{\prime}\right)\right]^{2} \\\left|g_{2, \delta}(x)-g_{2, \delta}(y)\right|>\delta}} \frac{\delta}{|x-y|^{N+1}} d x d y \geq \mathbf{b}_{N, 1}\left[g\left(t_{2}, z^{\prime}\right)-g\left(t_{1}, z^{\prime}\right)\right]\left|Q^{\prime}\left(z^{\prime}\right)\right| .
$$

From (6.24) and (6.25), (6.21) holds.

From (6.18(ii)) and (6.21), we deduce that

$$
\begin{gathered}
\frac{\lim _{\delta \rightarrow 0}}{\substack{\left[\left(t_{1}, t_{2}\right) \times Q^{\prime}\left(x^{\prime}\right)\right]^{2} \\
\left|g_{\delta}(x)-g_{\delta}(y)\right|>\delta}} \frac{\delta}{|x-y|^{N+1}} d x d y \geq \mathbf{b}_{N, 1}[1-2 \tau] \int_{Q^{\prime}\left(x^{\prime}\right)}\left|g\left(t_{2}, x^{\prime}\right)-g\left(t_{1}, x^{\prime}\right)\right| d x^{\prime} \\
-C \tau\left|Q^{\prime}\left(x^{\prime}\right)\right|
\end{gathered}
$$

Applying Besicovitch's covering theorem, it follows from (6.26) that

$$
\begin{aligned}
& \frac{\lim _{\delta \rightarrow 0}}{\substack{\left[\left(t_{1}, t_{2}\right) \times \Pi_{i=2}^{N}\left(a_{i}, b_{i}\right)\right]^{2} \\
\left|g_{\delta}(x)-g_{\delta}(y)\right|>\delta}} \frac{\delta}{|x-y|^{N+1}} d x d y \\
& \geq \mathbf{b}_{N, 1}[1-2 \tau] \int_{\Pi_{i=2}^{N}\left[a_{i}, b_{i}\right]}\left|g\left(t_{2}, x^{\prime}\right)-g\left(t_{1}, x^{\prime}\right)\right| d x^{\prime}-C \tau \Pi_{i=2}^{N}\left(b_{i}-a_{i}\right) .
\end{aligned}
$$

Since $\tau>0$ is arbitrary, we obtain the conclusion. 


\section{Remark 4}

It is surprising that the inequality in Lemma 14 involves the constant $C_{N, 1}$ (since $\mathbf{b}_{N, 1}=C_{N, 1}$ ), although $C_{N, 1}$ is defined by a process depending on a smooth function.

\subsection{Proof of Proposition 2 in the case $p=1$}

We recall that for each $g \in \mathrm{BV}\left(\mathbb{R}^{N}\right),\|D g\|$ is a Radon measure on $\mathbb{R}^{N}$, and there exists a $\|D g\|$-measurable function $\sigma: \mathbb{R}^{N} \mapsto \mathbb{R}^{N}$ such that

$$
\left\{\begin{array}{l}
|\sigma(x)|=1\|D g\| \text { a.e., } \\
\int_{\mathbb{R}^{N}} f \operatorname{div} \psi=-\int_{\mathbb{R}^{N}} \psi \cdot \sigma d\|D g\|, \quad \forall \psi \in C_{\mathrm{c}}^{1}\left(\mathbb{R}^{N} ; \mathbb{R}^{N}\right)
\end{array}\right.
$$

(see, e.g., [12, Theorem 1, p. 167]). Then for $\|D g\|$ a.e. $x \in \mathbb{R}^{N}$, we have (see, e.g., [12, Theorem 1, p. 38])

$$
\lim _{r \rightarrow 0} \frac{\|D g \cdot \sigma(x)\|(Q(x, \sigma(x), r))}{\|D g\|(Q(x, \sigma(x), r))}=1 .
$$

Hereafter for any $(x, \sigma, r) \in \mathbb{R}^{N} \times \mathbb{S}^{N-1} \times(0,+\infty), Q(x, \sigma, r)$ denotes the closed cube centered at $x$ with edge length $2 r$ such that one of its faces is orthogonal to $\sigma$.

Fix $\varepsilon>0$ (arbitrary). By Besicovitch's covering theorem, there exists a family of cubes

$\left(Q\left(x_{i}, \sigma\left(x_{i}\right), r_{i}\right)\right)_{i \in \mathbb{N}}$ such that

$$
\begin{gathered}
Q\left(x_{i}, \sigma\left(x_{i}\right), r_{i}\right) \cap Q\left(x_{j}, \sigma\left(x_{j}\right), r_{j}\right)=\varnothing \text { for } i \neq j, \\
\frac{\left\|D g \cdot \sigma\left(x_{i}\right)\right\|\left(Q\left(x_{i}, \sigma\left(x_{i}\right), r_{i}\right)\right)}{\|D g\|\left(Q\left(x_{i}, \sigma\left(x_{i}\right), r_{i}\right)\right)} \geq 1-\varepsilon, \\
\left\|D g \cdot \sigma\left(x_{i}\right)\right\|\left(\partial Q\left(x_{i}, \sigma\left(x_{i}\right), r_{i}\right)\right)=0,
\end{gathered}
$$

and

$$
\|D g\|\left(\mathbb{R}^{N}\right)=\|D g\|\left(\bigcup_{i \in \mathbb{N}} Q\left(x_{i}, \sigma\left(x_{i}\right), r_{i}\right)\right) .
$$

Hence it follows from (6.29) and (6.31) that

$$
\|D g\|\left(\mathbb{R}^{N}\right) \leq \frac{1}{1-\varepsilon} \sum_{i \in \mathbb{N}}\left\|D g \cdot \sigma\left(x_{i}\right)\right\|\left(Q\left(x_{i}, \sigma\left(x_{i}\right), r_{i}\right)\right) .
$$


Applying Lemma 14 and Proposition 3, we deduce from (6.30) that

$$
\mathbf{b}_{N, 1}\left\|D g \cdot \sigma\left(x_{i}\right)\right\|\left(Q\left(x_{i}, \sigma\left(x_{i}\right), r_{i}\right)\right) \leq \frac{\lim _{\delta \rightarrow 0}}{\substack{\left[Q\left(x_{i}, \sigma\left(x_{i}\right), r_{i}\right)\right]^{2} \\\left|g_{\delta}(x)-g_{\delta}(y)\right|>\delta}} \frac{\delta}{|x-y|^{N+1}} d x d y .
$$

Combining (6.32) and (6.33) yields

$$
\mathbf{b}_{N, 1}\|D g\|\left(\mathbb{R}^{N}\right) \leq \frac{1}{1-\varepsilon} \frac{\lim _{\delta \rightarrow 0}}{\substack{\left[\bigcup_{i=1}^{k} \\\left|g_{\delta}(x)-g_{\delta}(y)\right|>\delta\right.}} \underset{\int}{ } \frac{\delta}{|x-y|^{N+1}} d x d y .
$$

Since $\varepsilon>0$ is arbitrary and $\mathbf{b}_{N, 1}=C_{N, 1}$ (see Proposition 4), it follows from (6.34) that

$$
\varliminf_{\delta \rightarrow 0} I_{\delta}\left(g_{\delta}\right) \geq I(g)
$$

Acknowledgments. The author warmly thanks J. Bourgain for the discussion during the preparation of the paper [4]. The main ideas in the proof of Proposition 1 originate in [4]. He is deeply grateful to H. Brezis for conjecturing Theorem 2 and for his encouragement. He also thanks A. Ponce for communicating the example mentioned after Theorem 1 and V. Millot for interesting discussions. Part of this work was done when the author visited the Institute for Advanced Study and Rutgers University; he thanks these mathematics departments for their hospitality. The author also thanks the referees who carefully read the paper and made useful comments and suggestions which helped him improve the presentation of the paper and the proof of Proposition 2 in the case $p=1$.

\section{References}

[1] L. AMBROSIO, N. FUSCO, and D. PALLARA, Functions of Bounded Variation and Free Discontinuity Problems, Oxford Math. Monogr., Clarendon, Oxford Univ. Press, New York, 2000. MR 1857292

[2] J. BOURGAIN, H. BREZIS, and P. MIRONESCU, "Another look at Sobolev spaces" in Optimal Control and Partial Differential Equations: In Honour of Professor Alain Bensoussan's 60th birthday, IOS, Amsterdam, 2001, 439-455.

[3] J. BOURGAIN, H. BREZIS, and H.-M. NGUYEN, A new estimate for the topological degree, C. R. Math. Acad. Sci. Paris 340 (2005), 787-791. MR 2139888

[4] J. BOURGAIN and H.-M. NGUYEN, A new characterization of Sobolev spaces, C. R. Math. Acad. Sci. Paris 343 (2006), 75 - 80. MR 2242035 
[5] A. BRAIDES, $\Gamma$-Convergence for Beginners, Oxford Lecture Ser. Math. Appl. 22, Oxford Univ. Press, Oxford, 2002. MR 1968440

[6] H. BREZIS, How to recognize constant functions: A connections with Sobolev spaces (in Russian), volume in honor of M. Vishik, Uspekhi Mat. Nauk 57 (2002), no. 4, 59-74; English translation in Russian Math. Surveys 57 (2002), 693 - 708. MR 1942116

[7] - "New questions related to the topological degree" in The Unity of Mathematics: In Honor of the Ninetieth Birthday of I. M Gelfand, Progr. Math.

244, Birkhauser, Boston, 2006, 137-154. MR 2181804

[8] - private communication, 2005.

[9] H. BREZIS and H-M. NGUYEN, On the distributional Jacobian of maps from $\mathbb{S}^{N}$ into $\mathbb{S}^{N}$ in fractional Sobolev and Hölder spaces, to appear in Ann. of Math.

[10] D. CHIRON, On the definitions of Sobolev and BV spaces into singular spaces and the trace problem, Commun. Contemp. Math. 9 (2007), 473-513. MR 2348841

[11] G. DAL MASO, An introduction to $\Gamma$-convergence, Progr. Nonlinear Differential Equations Appl. 8, Birkhauser, Boston, 1993. MR 1201152

[12] L. C. EVANS and R. F. GARIEPY, Measure Theory and Fine Properties of Functions, Stud. Adv. Math., CRC, Boca Raton, Fla, 1992. MR 1158660

[13] H.-M. NGUYEN, Some new characterizations of Sobolev spaces, J. Funct. Anal. 237 (2006), 689-720. MR 2230356

[14] - Г-convergence and Sobolev norms, C. R. Math. Acad. Sci. Paris 345 (2007), 679-684. MR 2376638

[15] - Optimal constant in a new estimate for the degree, J. Anal. Math. 101 (2007), 367 -395. MR 2346551

[16] - Further characterizations of Sobolev spaces, J. Eur. Math. Soc. 10 (2008), 191 -229. MR 2349901

[17] - Some inequalities related to Sobolev norms, to appear in Calc. Var. Partial Differential Equations.

[18] A. PONCE, private communication, 2006.

[19] W. RUDIN, Real and Complex Analysis, 3rd ed., McGraw-Hill Ser. Higher Math., McGraw-Hill, New York, 1987. MR 0924157

[20] M. STEIN, Singular Integrals and Differentiability Properties of Functions, Princeton Math. Ser. 30, Princeton Univ. Press, Princeton, 1970. MR 0290095

Courant Institute, New York University, New York, New York 10012, USA;

hoaiminh@cims.nyu.edu 
